\title{
Comparison of surface and volume integral methods for transonic propeller acoustic predictions
}

\author{
G. Rahier ${ }^{1}$ \\ ONERA - The French Aerospace Lab, Châtillon, F-92322, France \\ Corresponding author: gilles.rahier@ onera.fr \\ Tel: +33146734812 \\ Fax: +33146734166
}

\begin{abstract}
A precise comparison of the surface and volume approaches of the Ffowcs Williams-Hawkings acoustic integral formulation is conducted for a propeller in transonic operating conditions. For both approaches, the calculations are carried out directly starting from CFD input data provided in the propeller rotating frame, i.e. with supersonically moving emission points. No approximation in the volume calculations, which could distort the comparison between both methods, is made. The principle and the calculation algorithm on which this particular integration technique is based are reviewed. Then calculations are carried out for four increasingly refined CFD meshes. They first confirm that both acoustic integral methods provide identical results when the numerical dissipation is negligible in the aerodynamic calculation. These calculations also show that the volume method is slightly less sensitive to the numerical dissipation than the surface method. However, the gain seems low compared to the computational cost of the volume integration. In addition, two techniques for determining the regions of the dominant acoustic sources are explored. With the first one, a rather conventional technique based on the local quadrupole term, the results show that specific terms, chosen according to the phenomenon concerned, may be better indicators of the real noise sources than the original shear and entropy terms. The second one, less known and consisting in calculating the elementary acoustic time signature radiated by each cell of the grid, seems more effective but may turn out to be costly in terms of data storage with the volume method.
\end{abstract}

Keywords: computational aeroacoustics, acoustic integral methods, Ffowcs Williams-Hawkings, quadrupolar noise, transonic rotors

\footnotetext{
${ }^{1}$ Research Scientist at Aerodynamics Aeroelasticity Acoustics Department
} 


\section{List of symbols}

$a_{0} \quad$ Speed of sound in the unperturbed medium

$e_{i j} \quad$ Viscous stress tensor

$M_{0}$ Mach number of the unperturbed fluid in the reference frame $\boldsymbol{Y}$

$\vec{n} \quad$ Normal unit vector on the control surface, pointing outward

p Local pressure

$p_{0} \quad$ Pressure in the unperturbed fluid

$p^{\prime}=p-p_{0}$ Pressure perturbation

$t \quad$ Reception time

$T_{i j} \quad$ Lighthill stress tensor

$U_{0} \quad$ Velocity of the unperturbed fluid in the reference frame $\boldsymbol{Y}$

$\vec{u} \quad$ Local fluid velocity in the reference frame $y$

$\vec{U} \quad$ Local fluid velocity in the reference frame $\boldsymbol{Y}$

$U_{n}=\vec{U} \cdot \vec{n}=U_{i} n_{i}$ Fluid velocity component normal to the control surface

$\vec{v} \quad$ Local control surface velocity in the reference frame $y$

$\vec{V} \quad$ Local control surface velocity in the reference frame $\boldsymbol{Y}$

$v_{n}=\vec{v} \cdot \vec{n}=v_{i} n_{i} \quad$ Surface velocity component normal to the control surface

$\vec{X} \quad$ Observer location in the reference frame $\boldsymbol{Y}$

$\boldsymbol{y} \quad$ Reference frame in which the unperturbed fluid is at rest

$\boldsymbol{Y}$ Reference frame in which the unperturbed fluid is moving at the uniform velocity $U_{0} \vec{y}_{1}$

$\vec{Y} \quad$ Source location in the reference frame $\boldsymbol{Y}$

$\delta(f)$ Dirac distribution

$\delta_{i j} \quad$ Kronecker symbol

$\rho \quad$ Density 


\section{$\rho_{0} \quad$ Density of the unperturbed fluid \\ $\rho^{\prime}=\rho-\rho_{0} \quad$ Density perturbation}

$\tau \quad$ Emission time

\section{Introduction}

The Ffowcs Williams-Hawkings (FW-H) formulation [1] is an effective tool for acoustic analysis of aerodynamic fields. This formulation generalises Lighthill's equation [2] by including solid or fictitious (porous) surfaces in the flow [3]. In its complete form, it provides the acoustic far-field radiation of flow disturbances by simply performing surface and volume integrals on quantities that can be provided in near field by a CFD computation. It is worth mentioning that the assumptions of a uniform flow and of a free field outside the integration area are generally made in the Green function used for the radiation calculation.

In some cases (e.g. a body in an almost uniform low speed flow, with low turbulence), the volume sources (in the sense of Lighthill) can be neglected and the acoustic calculation is limited, in practice, to the integration of source terms on the solid surface of the body. When the volume sources cannot be neglected (turbulent jets or transonic blades with shocks, for example), acoustic calculations using only surface integrals (on porous surfaces surrounding the noise sources) can nevertheless be performed and are preferred to volume calculations for obvious reasons of amount of input data and computational cost. However, the porous surface method, theoretically equivalent to the volume method, has two drawbacks.

- The first drawback relates to the fact that the integration surface (the control surface) must encompass all phenomena involved in the acoustic radiation such as nonlinear propagations effects (stiffening or damping of a shock, for example) or the effects of the non-uniformity of the flow in the vicinity of the noise sources (refraction effects). Therefore, the control surface must be sufficiently far from the primary noise sources in order to be in a region of linear propagation and in a virtually uniform flow. This is a major constraint for the CFD calculations which must then carry the flow disturbances up to the control surface, with a minimal numerical dissipation and dispersion.

- The second drawback is more specific to the surface approach: the surface integration does not provide direct information on the spatial distribution of the noise sources, since the aerodynamic fields on the 
control surface result from all phenomena that are present in the inside volume. However, the location of noise sources is possible by using microphone arrays [4], but this process increases the cost of the acoustic calculation by multiplying the number of observation points in the far field, and introduces biases (due to the source and propagation models) specific to the method of localization. Other methods of source localization, within the broader framework of solving the inverse problem, are subject to numerous studies (a synthesis of these studies can be found in [5]), but their maturity still seems insufficient for application to non-academic configurations.

Concerning the first point, one may wonder whether the volume method is less sensitive to numerical dissipation in the aerodynamic calculation than the surface method, and could thus reduce the constraints on CFD computations regarding the transportation of perturbations. In other words, in the presence of significant numerical dissipation, does the integration of source terms in a volume provide better results than the integration of source terms on the boundary of this volume? This study aims at answering this issue in the case of high speed impulsive (HSI) noise generated by a propeller in transonic operating conditions. The analysis is mainly based on the comparison of the results provided by both surface and volume approaches, for various levels of dissipation in the CFD calculation. To our knowledge, such a parametric study has never been done, because the usual volume integration techniques include simplifications that would distort this analysis (observer in the far field and on the rotor plane, approximate volume sources [6][7][8], volume sources neglected beyond the sonic cylinder [6]) or would be too expensive without these simplifications. The fully non-compact integration technique developed at ONERA, which can directly deal with supersonically moving meshes [9][10] without any simplification of the volume integral, makes it possible to compare the surface and volume methods rigorously. Calculations are performed for an isolated propeller with a zero angle of attack, which also makes acoustic calculations easier. Indeed, the aerodynamic fields are then steady in the rotating frame of the blades and the amount of CFD input data remains moderate (a few tens of megabytes to several gigabytes depending on the extent and the density of the grid).

In addition to this parametric study, volume calculations are used to answer the second point by exploring two acoustic source localization techniques.

The article is organized as follows. The expressions of the surface and volume integrals and the numerical method used to calculate them with supersonic rotating grids are first reviewed in Section 2. The case studied and the numerical setup are described in Section 3. The results are then presented and discussed in Section 4. 


\section{Acoustic formulation and integration technique}

\section{$2.1 \quad F W$-H formulation}

The framework of the present acoustic calculations is as follows: $\boldsymbol{y}$ is a reference frame in which the unperturbed fluid is at rest, $\boldsymbol{Y}$ is a reference frame in translation at the velocity $-U_{0} \vec{y}_{1}$ with respect to $\boldsymbol{y}, \boldsymbol{S}$ is a closed moving surface permeable to the fluid, and $\boldsymbol{V}$ a bounded volume outside the surface $\boldsymbol{S}$. Any solid body interacting with the fluid is assumed to be inside $S$ and the acoustic sources as defined by Lighthill (double divergence of the Lighthill tensor $T_{i j}$, see below) are supposed negligible beyond the volume $\boldsymbol{V}$.

Starting from the extension to porous surfaces [3][11] of the Ffowcs Williams and Hawkings formulation [1], using the space coordinates $Y_{i}$ in the reference frame $\boldsymbol{Y}$ and the velocities $\vec{u}$ of the fluid and $\vec{v}$ of the surface $\boldsymbol{S}$ in the reference frame $\boldsymbol{y}$, the pressure received by observer at location $\vec{X}$ and time $t$ can be written as:

$$
p^{\prime}(\vec{X}, t)=-\frac{\partial}{\partial X_{i}} \int_{\tau} \int_{S} A_{i} G d S d \tau+\frac{d}{d t} \iint_{\tau} B G d S d \tau+\frac{\partial^{2}}{\partial X_{i} \partial X_{j}} \int_{\tau} \int_{V} T_{i j} G d V d \tau
$$

where $\quad T_{i j}=\rho u_{i} u_{j}+\left(p^{\prime}-a_{0}^{2} \rho^{\prime}\right) \delta_{i j}-e_{i j}, \quad A_{i}=p^{\prime} n_{i}-e_{i j} n_{j}+\rho u_{i}\left(u_{n}-v_{n}\right), \quad B=\rho_{0} v_{n}+\rho\left(u_{n}-v_{n}\right)$, $\frac{d}{d \tau}=\left(\frac{\partial}{\partial \tau}\right)_{Y}+U_{0} \frac{\partial}{\partial Y_{1}}$ and $G=\delta(g) / 4 \pi d$ the free-space Green's function in $R_{3}$ for the convective wave equation with $g=\tau-t+\sigma / a_{0}, \sigma=\left(d-M_{0}\left(X_{1}-Y_{1}\right)\right) / \beta^{2}, d=\sqrt{C_{i}\left(X_{i}-Y_{i}\right)^{2}}, C_{i}=\left(1-\beta^{2}\right) \delta_{1 i}+\beta^{2}, \beta^{2}=1-M_{0}^{2}$, and $M_{0}=U_{0} / a_{0}$ (see details in [12]).

In the present study, as is commonly done, the viscous term $e_{i j}$ is ignored.

\subsubsection{Formula used for surface integrals}

Both surface integrals of formula (1) can be transformed to avoid the calculation of the spatial derivatives $\partial / \partial X_{i}$, and thus avoid acoustic calculation for numerous observation points around $\vec{X}$ (six points for usual centered derivatives by finite differences). They can write [13]: 


$$
\begin{aligned}
p_{S}^{\prime}(\vec{X}, t) & \left.=\int_{\tau} \int_{S} A_{i} \frac{\partial d}{\partial X_{i}}-B U_{0} \frac{\partial d}{\partial X_{1}}\right) \frac{\delta(g)}{4 \pi d^{2}} d S d \tau \\
& +\frac{\partial}{\partial t} \int_{\tau} \int_{S}\left(A_{i} \frac{\partial g}{\partial X_{i}}-B U_{0} \frac{\partial g}{\partial X_{1}}+B\right) \frac{\delta(g)}{4 \pi d} d S d \tau
\end{aligned}
$$

Remark: An analytical time integration of (2) is generally performed by changing the integration variable $\tau$ in $g$, which leads to the following formula:

$$
\begin{aligned}
p_{S}^{\prime}(\vec{X}, t) & \left.=\int_{S}\left[\int_{i} \frac{\partial d}{\partial X_{i}}-B U_{0} \frac{\partial d}{\partial X_{1}}\right) \frac{1}{4 \pi d^{2}|\partial g / \partial \tau|}\right]_{g=0} d S \\
& +\frac{\partial}{\partial t} \int_{S}\left[\left(A_{i} \frac{\partial g}{\partial X_{i}}-B U_{0} \frac{\partial g}{\partial X_{1}}+B\right) \frac{1}{4 \pi d|\partial g / \partial \tau|}\right]_{g=0} d S
\end{aligned}
$$

where the quantities inside the brackets are evaluated at the emission time $\tau=t-\sigma / a_{0}$. For a surface $S$ in subsonic motion relative to the observation point $\vec{X}, \partial g / \partial \tau$ is never zero and the usual locally compact integration technique ( $\boldsymbol{S}$ discretized in source elements $\delta S_{i}$ treated as point sources) then allows a rather easy calculation of the radiated pressure. Formula (2bis) is still mathematically valid for a surface $\boldsymbol{S}$ in transonic relative motion, but it will not be used in the present study. The numerical integrations will be carried out by restarting from formula (2), for reasons discussed in Section 2.2.

\subsubsection{Formula used for volume integrals}

In the same way as for the surface integrals, the volume integral of formula (1) can also be transformed to avoid spatial derivation of the radiated pressure or of the aerodynamic fields and can write [12]:

$$
\begin{aligned}
p_{V}^{\prime}(\vec{X}, t) & =\int_{\tau} \int_{V} T_{i j}\left(\frac{2}{d} \frac{\partial d}{\partial X_{i}} \frac{\partial d}{\partial X_{j}}-\frac{\partial^{2} d}{\partial X_{i} \partial X_{j}}\right) \frac{\delta(g)}{4 \pi d^{2}} d V d \tau \\
& +\frac{\partial}{\partial t} \int_{\tau} \int_{V} T_{i j}\left[\frac{1}{d}\left(\frac{\partial d}{\partial X_{i}} \frac{\partial g}{\partial X_{j}}+\frac{\partial g}{\partial X_{i}} \frac{\partial d}{\partial X_{j}}\right)-\frac{\partial^{2} g}{\partial X_{i} \partial X_{j}}\right] \frac{\delta(g)}{4 \pi d} d V d \tau
\end{aligned}
$$




$$
+\frac{\partial^{2}}{\partial t^{2}} \int_{\tau} \int_{V} T_{i j} \frac{\partial g}{\partial X_{i}} \frac{\partial g}{\partial X_{j}} \frac{\delta(g)}{4 \pi d} d V d \tau
$$

The previous remark concerning analytical time integration for surface integrals also applies to volume integrals. For the latter, this analytical time integration leads to the following expression (not used in the present study, see Section 2.2).

$$
\begin{aligned}
p_{V}^{\prime}(\vec{X}, t) & =\int_{V}\left[\frac{T_{i j}}{4 \pi d^{2}}\left(\frac{2}{d} \frac{\partial d}{\partial X_{i}} \frac{\partial d}{\partial X_{j}}-\frac{\partial^{2} d}{\partial X_{i} \partial X_{j}}\right) \frac{1}{\partial g / \partial \tau \mid}\right]_{g=0} d V \\
& +\frac{\partial}{\partial t} \int_{V}\left[\frac{T_{i j}}{4 \pi d}\left(\frac{1}{d}\left(\frac{\partial d}{\partial X_{i}} \frac{\partial g}{\partial X_{j}}+\frac{\partial g}{\partial X_{i}} \frac{\partial d}{\partial X_{j}}\right)-\frac{\partial^{2} g}{\partial X_{i} \partial X_{j}}\right) \frac{1}{\partial g / \partial \tau \mid}\right]_{g=0} d V \\
& +\frac{\partial^{2}}{\partial t^{2}} \int_{V}\left[\frac{T_{i j}}{4 \pi d} \frac{\partial g}{\partial X_{i}} \frac{\partial g}{\partial X_{j}} \frac{1}{\partial g / \partial \tau}\right]_{g=0} d V
\end{aligned}
$$

\subsection{Integration technique}

As mentioned in the introduction, in the case of stationary fields in a rotating frame, the volume of input data to be stored for acoustic post-processing by a volume method is not exactly a difficulty. If acoustic calculations are carried out directly using the CFD rotating mesh (as is done in the present study), only aerodynamic fields for a reference emission time $\tau_{0}$ need to be stored. In fact, the difficulty for transonic configurations lies in the integration technique. Indeed, the noise sources related to the shocks extend radially beyond the sonic cylinder (cylinder on which the relative Mach number is equal to 1) and the integrals in formulas (2bis) and (3bis) can then be singular beyond the sonic cylinder ( $\partial g / \partial \tau=0$ for some observer locations). An example of the radial extent of noise sources is shown in Fig. 1 for a delocalized test case of the non-lifting UH1H model rotor in hover [14] (aerodynamic input data generated for study [15] by J. Baeder running the TURNS code [16] in the Euler mode and acoustic calculations performed in [17]). In particular, it shows the strong contribution of volume sources outside the sonic cylinder to the radiated pressure, and therfore the need to take them into account in acoustic calculations. 

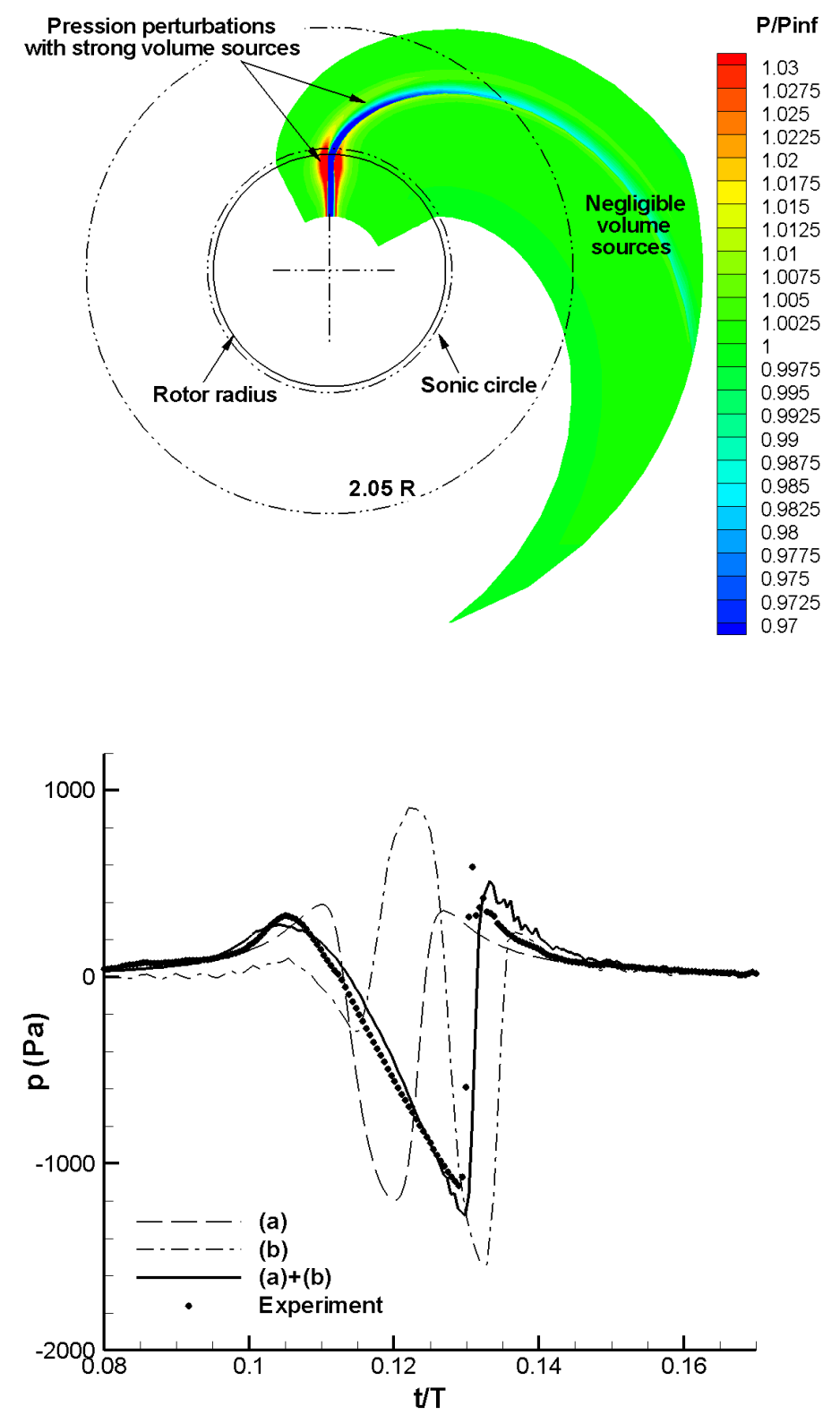

Fig. 1. UH1H model rotor in hover, tip Mach number $=0.95$. Pressure distribution in the rotor plane (top) and contributions to the acoustic time signature at $3.09 \mathrm{R}$ in the rotor plane (bottom):

(a) sources inside the sonic cylinder, (b) sources outside the sonic cylinder. 
A solution, when using the usual integration techniques that cannot deal with the singularity $\partial g / \partial \tau=0$, is to perform a projection of the rotating CFD fields onto a fixed grid (e.g. [18]). However, this projection must be made on a relatively fine uniform grid to properly capture shocks during their rotation, at each step of a relatively fine time discretization, for a good description of the shocks' kinematic. This projection may turn out to be a very costly operation as well as a source of imprecisions in the input data of the acoustic calculations.

For surface methods, other integration procedures have been developped to solve the numerical problem raised by this singularity (collapsing sphere formulation [19][20], emission surface algorithms [20][21][22]). However, given their computational cost, they seem challenging to apply to volume methods. An integration technique that is, from our point of view, much more efficient, has previously been developped at ONERA, for surface methods [9][13][17]. This fully non-compact integration technique is applied here to both surface and volume calculations. The principle of this original approach (described in detail in [9][10]) is reviewed below.

The singularity appearing with supersonic moving meshes is purely numerical. It is due to the modeling of each cell of the mesh by a point source (locally compact approach of the usual integration techniques). This model does not reflect the continuity of the integration domain, especially the fact that at a given emission time, the different points of a cell are not all at the same distance from the observer. In fact, because of these different distances, the emission of a cell at time $\tau$ is not received at a single instant $t$, but during a time interval $\left[t_{1}, t_{2}\right]$ whose boundaries depend on the size, shape and orientation of the cell.

The locally compact approach leads to focusing the radiation of the whole cell onto the same reception time. This phenomenon of acoustic focusing is accentuated when the velocity of the source point towards the observation point tends to the speed of sound. The radiation then "piles up" even more on the same reception time, which explains the divergence in the results for the transonic moving meshes. It should be noted that the refinement of the mesh or of the time step tends to further degrade the results by increasing the number of source points moving at a velocity close to the speed of sound towards the observation point.

A relatively simple way of taking into account the continuity of the integration domain consists in, rather than modeling the emission of the cell at the time $\tau$ by a point source, modeling the reception of the radiated signal at the observation point by a pressure rate $\Pi$ (in $\mathrm{Pa} / \mathrm{s}$ ) over the time interval $\left[t_{1}, t_{2}\right]$. This is done in the so-called "fully noncompact" integration technique which does not use the analytical time integration leading to expressions (2bis) and (3bis) but restarts from formulas (2) and (3). 
In practice, the reception intervals $\left[t_{1}, t_{2}\right]$ are determined for each cell $\Delta_{\mathrm{i}}$ of the integration domain and for each emission time $\tau_{j}$, starting from the position of the observation point and the position of the nodes of the cell at time $\tau_{j}$. The associated radiated pressure rate $\Pi_{i j}$ is calculated as a function of the considered source term $F$ (which can be evaluated at the center of the cell), of the surface or volume $\left|\Delta_{\mathrm{i}}\right|$ of the cell, and of the reception time duration $t_{2}-t_{1}$. For instance, if a constant pressure rate is considered, the pressure rate $\Pi_{i j}$ is then equal to $F \mid \Delta_{\mathrm{i}} /\left(t_{2}-t_{1}\right)$ over the time interval $\left[t_{1}, t_{2}\right]$ and to zero outside. The pressure received at the observation point at a given instant $t$ is then determined by summing this pressure rate over the emission times (see Fig. 2). This time integration can be quite simple (depending on the pressure rate model), since it consists in calculating the area of the hatched surface in Fig. 2 by linear interpolations starting from the reception time intervals and the pressure rates determined for the emission times $\tau_{j}$ and $\tau_{j+1}$. The space integration is then carried out by summing the pressure contributions of each cell of the mesh. It is worth pointing out that this integration technique gives the same result as the usual locally compact method when the mesh is fixed. This fully non-compact approach has been thoroughly validated [9] and is used by ONERA and by its industrial partners, for rotors and propellers acoustic studies (for example [23]-[26]).

To facilitate the calculation procedure, a pressure rate that is constant over the reception interval, and therefore discontinuous at the boundaries of this interval, was first used (Fig. 2, top). This very simple model has given full satisfaction for formulations without time derivatives outside the integral [10]. A continuous increasing/decreasing model (Fig. 2, bottom) has then been designed for formulations with time derivatives outside the integral, which require more regular results for these time derivations. In this study, all acoustic calculations are carried out using this second model. 

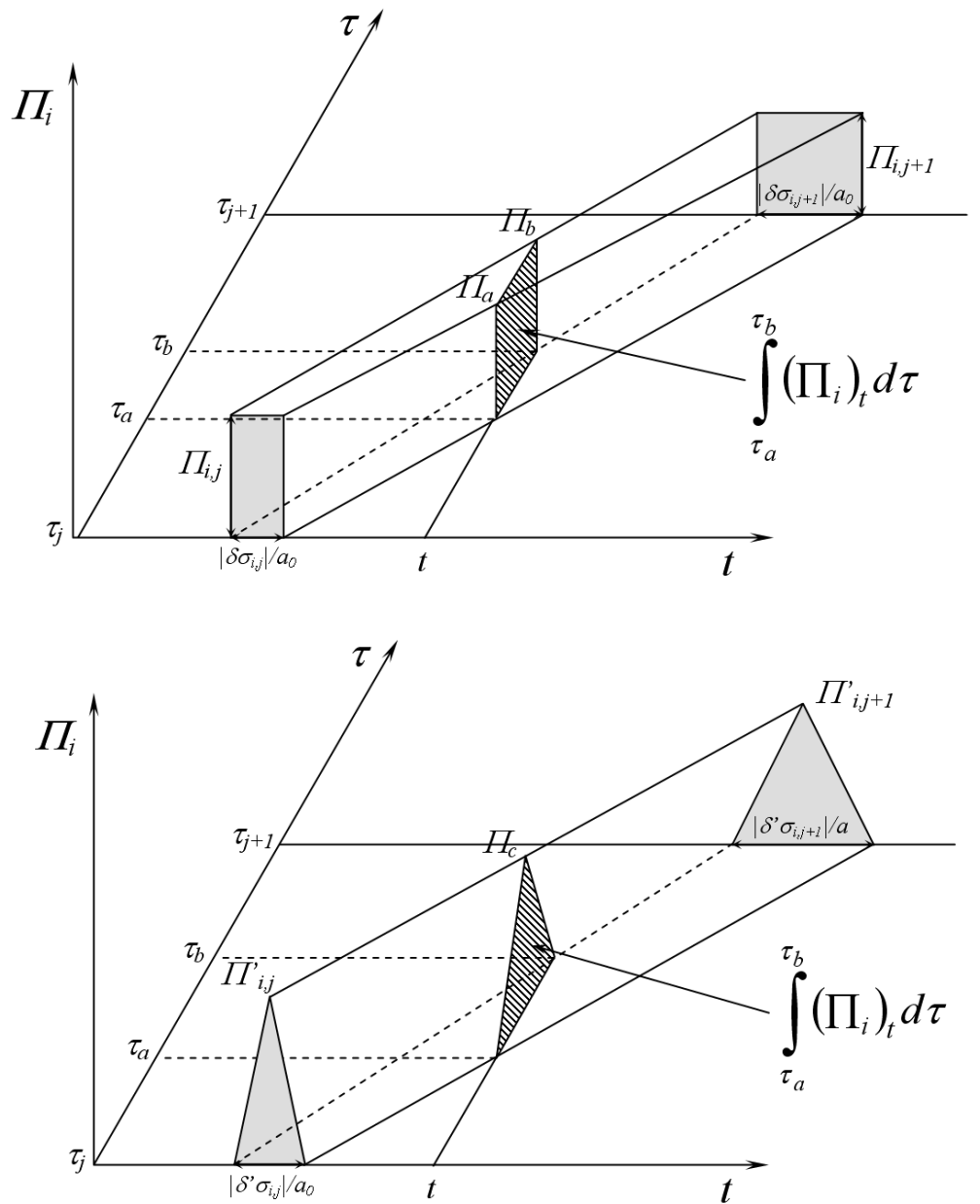

Fig. 2. Schematic of the time integration process for a non-compact source element, for two pressure rate shape functions: rectangular (top) and triangular (bottom).

\section{Case studied and numerical setup}

\subsection{Configuration and CFD input data}

Acoustic calculations are performed for an isolated propeller mounted on a nacelle (Fig. 3), with a zero angle of attack, so that the aerodynamic fields are steady in the rotating frame of the propeller. This configuration is that of the AIPX7 counter-rotating open rotor (geometry designed by Airbus for Clean Sky European project), in which the rear rotor has been removed. This is the same configuration as the one considered in [27] for numerical error analysis in the aeroacoustic simulation of transonic propellers. This previous study relied particularly on the 
aerodynamic fields provided by CFD simulations, carried out with meshes of different densities (thus leading to different levels of accuracy), on one hand, and on acoustic calculations performed using a surface method, on the other hand. The same aerodynamic fields are used here for the analysis of the volume method. The configuration, meshing technique, grid refinement and CFD computations are briefly described here. More details can be found in [27].

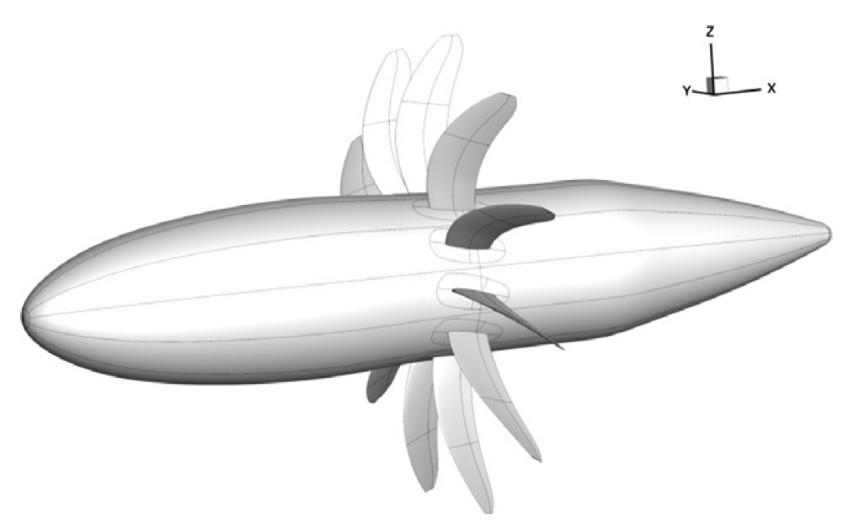

Fig. 3. Study configuration (picture extracted from [27]).

The radius $R$ of this eleven-bladed rotor is $2.15 \mathrm{~m}$. The operating conditions are: free-stream Mach number of 0.73, rotational tip Mach number of 0.6 , static pressure and speed of sound at infinity respectively equal to 23,837 $\mathrm{Pa}$ and $296.95 \mathrm{~m} / \mathrm{s}$. The helical Mach number is equal to 0.945 . This configuration, representative of cruise conditions, clearly leads to a transonic flow with a strong contribution of volume sources related to the formation and propagation of the shocks generated by the blades.

The aerodynamic calculations take into account the spatial periodicity of the flow field and the computational domain is reduced to the angular sector of one blade. The CFD mesh consists of structured blocks in the near-body region and cylindrical structured blocks in the off-body region (Fig. 4), which has made the successive mesh refinements easier. It extends from $-5.23 R$ to $5.23 R$ in the axial direction, and up to $3.8 R$ in the radial direction. 

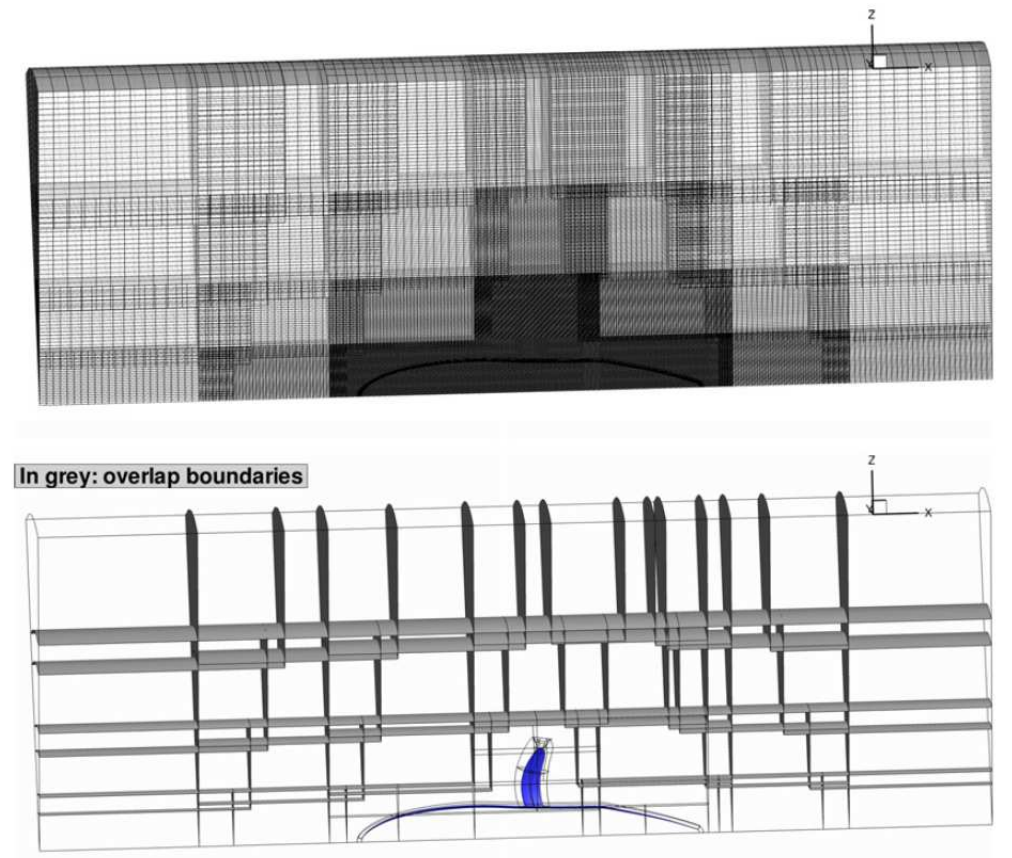

Fig. 4. CFD mesh (pictures extracted from [27]).

Starting from the coarsest one, the mesh is derived into 3 other versions. Each of them is built starting from the previous one by increasing the number of nodes of the background core blocks in all three space directions by a factor equal to 1.5 (except for the most refined mesh: 1.2). The near-field body grids are the same for all calculations. Table 1 states the total number of cells for each of the four meshes.

\section{Table 1}

Total number of cells for each CFD grid

\begin{tabular}{ccccc}
\hline CFD grid & Coarse & Medium & Fine & Super fine \\
Number of cells (in million) & 3.63 & 8.01 & 22.35 & 36.48 \\
\hline
\end{tabular}

The steady RANS equations are solved in the rotating frame of the blade, using a finite-volume solver [28] with a classic cell-centered Jameson scheme, second-order accurate in space. Interpolations between overset grids are second-order accurate in space. The turbulence model is Kok k- $\omega$ [29] with shear stress transport (SST) correction [30]. The solid surfaces of the body are modelled with a nonslip, adiabatic wall boundary condition. The outer boundaries of the computational domain are modelled with a far field boundary condition based on the characteristic 
relations. The input data of the acoustic calculations are the density, velocity and pressure provided at the nodes of the acoustic meshes described below.

\subsection{Surfaces and volumes of integration}

Some blocks of CFD meshes partially overlap. This is the case in the immediate vicinity of the blade and beyond a certain radial or axial distance from the blade. In these regions, these overlaps do not allow direct use of the CFD grid for acoustic calculations. They require the aerodynamic fields to be projected onto a new mesh defining the surface or the volume of integration. To avoid the construction of new meshes and the inaccuracies introduced by this projection, the volume of fluid under study is limited to the blocks near the blade that do not overlap. The number of blocks selected depends on the grid (see Table 2), however they all define the same volume of fluid. This volume is shown in Fig. 5 for the coarse grid and for the super fine grid.
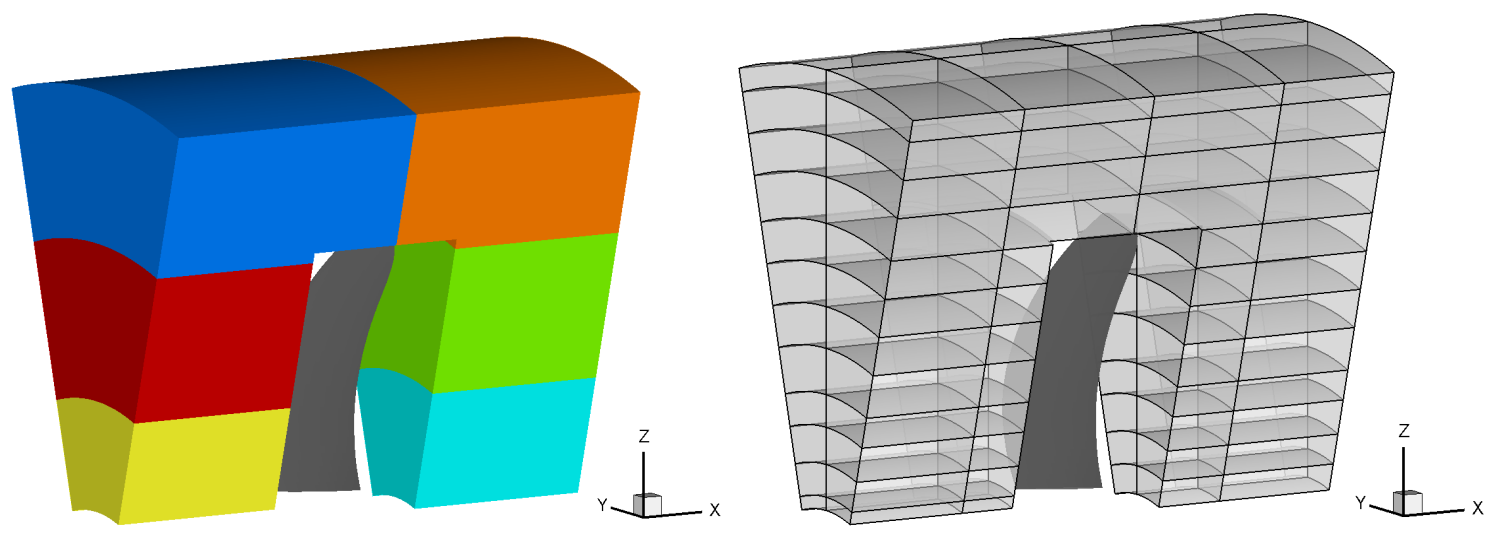

Fig. 5. Volume studied and blocks involved in the coarse CFD grid (left) and in the super fine CFD grid (right).

\section{Table 2}

Number of blocks involved and azimuthal discretization for acoustic calculation, for each CFD grid

\begin{tabular}{ccccc}
\hline CFD grid & Coarse & Medium & Fine & Super fine \\
Number of blocks involved & 6 & 17 & 48 & 96 \\
Azimuthal discretization & 45 & 65 & 98 & 114 \\
\hline
\end{tabular}


In this defined volume, ten control surfaces progressively enclosing more fluid around the blade are considered, in order to observe the contribution of volume sources and the effects of the CFD grid density on acoustic predictions (Fig. 6). These surfaces explore the same volume of fluid as that explored by the twenty control surfaces used by A. Giauque et al. in [27]. However, contrary to study [27] in which the axial and radial sizes of the control surfaces increase at the same time, here the radial extent of the control surfaces increases first (Fig. 6, S1 to S6), then the axial extent does (Fig. 6, S6 to $S 10$ ). This can help to distinguish the contribution of volume sources related to the formation and propagation of the shock (essentially in the radial direction) more easily from volume sources related to the blade wake. All these control surfaces have a cylindrical part (more or less extended) in their lower section, so that two consecutive surfaces constitute the closed boundary of the volume between them. This allows comparisons between surface and volume approaches, as will be shown in Section 4.

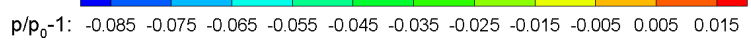
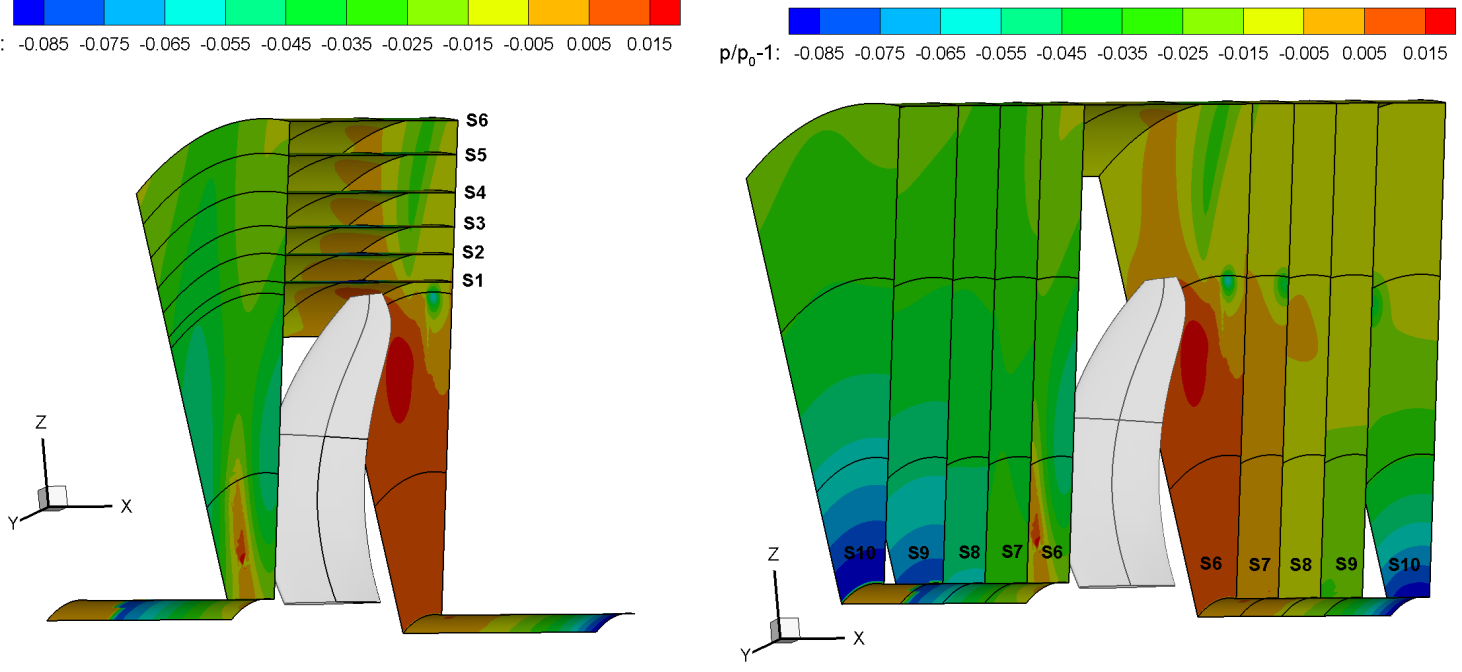

Fig. 6. Display of the different control surfaces (colored by the pressure perturbation) surrounding the blade (in grey).

The axial and radial extents of the different control surfaces (excluding their lower cylindrical parts) are given in Table 3. It can be noted that the volume under study extends beyond the sonic cylinder (helical Mach number equal to 1.103 on the outer radial boundary), which is not a difficulty for the integration method. Thereafter, the volume named $V i$ is the volume between $S 1$ and $S i(2 \leq \mathrm{i} \leq 10)$ and the volume $V A$ is the volume between the surfaces $S 6$ and S10. Table 4 gives the number of cells of the largest integration domains as a function of the grid, for the surface and volume methods. 
The usual process for taking volume sources into account in acoustic calculations is to add the result of the integration (3) in a volume of fluid around the body to that of the integration (2) on the body solid surface. In the present case, where the volume of fluid studied does not extend up to the blade, the body solid surface is replaced by the porous surface closest to the blade $(S 1)$. Therefore the analysis of the volume approach consists here in comparing $p_{S I}+p_{V i}$ to $p_{S i}$, where $p_{V i}$ is the radiated pressure calculated by integration in a given volume $V i$ and $p_{S i}$ that obtained by integration on the surface $S i$.

\section{Table 3}

Axial $(\Delta x)$ and radial $(r / R)$ extents of the control surfaces (excluding the lower cylindrical parts)

\begin{tabular}{ccccccccccc}
\hline Surface & $S 1$ & $S 2$ & $S 3$ & $S 4$ & $S 5$ & $S 6$ & $S 7$ & $S 8$ & $S 9$ & $S 10$ \\
$\Delta x(\mathrm{~m})$ & 0.8 & 0.8 & 0.8 & 0.8 & 0.8 & 0.8 & 1.2 & 1.6 & 2.0 & 2.6 \\
$r / R$ & 1.023 & 1.079 & 1.153 & 1.228 & 1.302 & 1.377 & 1.377 & 1.377 & 1.377 & 1.377 \\
\hline
\end{tabular}

\section{Table 4}

Maximum number of cells for the surface and for the volume acoustic calculations, depending on the grid

\begin{tabular}{ccccc}
\hline Grid & Coarse & Medium & Fine & Super fine \\
Surface $(S 10)$ & 15,975 & 35,396 & 78,498 & 111,264 \\
Volume $(V 10)$ & 337,140 & $1,142,998$ & $3,813,314$ & $6,502,902$ \\
\hline
\end{tabular}

\subsection{Calculation procedure}

Acoustic calculations must take all the blades into account in order to reproduce the interferences of their sound radiations. This can be done by an angular duplication of the CFD grid and the associated aerodynamic fields, but in the present case, it multiplies by 11 the volume of input data and the computation time. In fact, the angular periodicity of the flow-fields makes it possible to obtain the same result by adding to the signal radiated by a single blade 10 identical signals respectively time-shifted by (i-1)T/11 (i: blade number, T: rotation period of the propeller). Acoustic calculations are thus carried out by rotating the surface and the volume acoustic meshes described above, on a revolution. This rotation is described by 517 time steps (517 is a multiple of 11 , which makes the time shifting procedure mentioned previoulsy easier).

Surface and volume calculations are performed for 91 far-field observation points, distributed on a semi-circle of 
$8 \mathrm{~m}$ radius on either side of the propeller rotation plane (Fig. 7). Their angular position $\theta$ is defined with respect to the propeller rotation axis and ranges from $0 \mathrm{deg}$. (upstream) to $180 \mathrm{deg}$. (downstream).

Remark: For industrial confidentiality reasons, sound pressure levels (in $\mathrm{Pa}$ or in $\mathrm{dB}$ ) are hidden. The pressure scales, however, are identical for all the figures so that the different results can be compared.

The calculation times, on one processor running at $3 \mathrm{GHz}$, are $0.4410^{-6} \mathrm{~s} / \mathrm{cell} / \mathrm{time}$ step/obs. for surface calculations and $0.7210^{-6} \mathrm{~s} /$ cell/time step/obs. for volume calculations. Therefore, for 517 time steps and 91 observation points, surface calculations take between $6 \mathrm{~min}$ (coarse grid) and $40 \mathrm{~min}$ (super fine grid), and volume calculations take between 3 hours (coarse grid) and 61 hours (super fine grid). Therefore, volume calculations take 30 to 90 times longer than surface calculations, mainly because of the much higher number of integration cells. It should be noted that the parallelization of these calculations is very efficient (no information to be transmitted between cells, speed-up close to 1 or even greater than 1 since the memory access is faster with smaller arrays). Volume calculations with the super fine mesh, carried out on 64 processors, would thus probably take less than an hour.

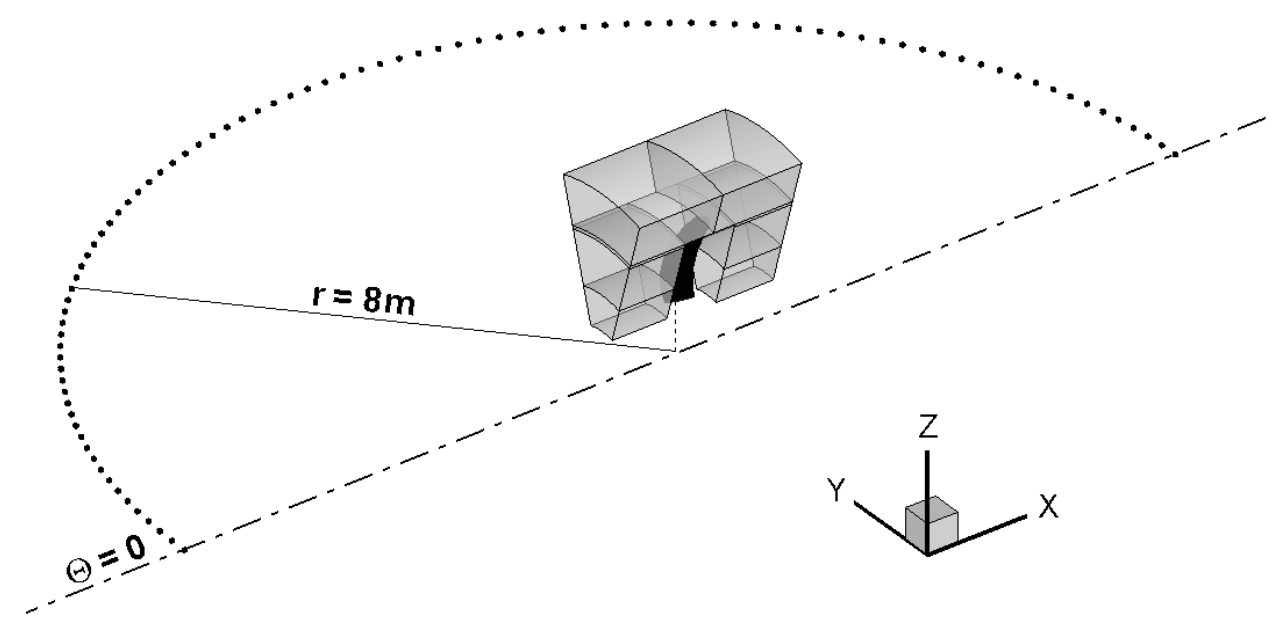

Fig. 7. Observation points used for the analysis of the propeller's acoustic radiation. 


\section{Results}

\subsection{Surface calculations}

An acoustic analysis of the aerodynamic fields is first performed using the surface method. Results will serve as reference for volume calculations. It should be noted that the analysis carried out in [27] is not resumed using other control surfaces. Surface calculations are only used here to evaluate the interest of the volume method by comparing the results.

The signals obtained in the propeller plane $(\theta=90$ deg. $)$, depending on the control surface, are plotted in Fig. 8 for the coarse grid and in Fig. 9 for the fine grid. For both grids, the amplitude and the asymmetry of the signal increase as the control surface increasingly encloses the volume sources related to the formation and propagation of the pressure perturbation (surfaces $S 1$ to $S 6$ ). The signals seem to stabilize for the control surfaces $S 5$ and $S 6$, which suggests that the surface $S 6$ encloses the bulk of the volume sources related to the shock. One can also observe a steeper recompression slope with the fine grid than with the coarse grid, which indicates a richer content in high frequency with the former. The amplitude and especially the shape of the signal do not change substantially when the control surface then extends in the axial direction (surfaces $S 6$ to $S 10$ ). The stability of the signal obtained with the fine grid (Fig. 9, right) tends to show the absence of significant volume sources in the blade wake (in the present case of RANS aerodynamic calculations). This means that the progressive decay of the amplitude of the negative pressure peak with the coarse grid (Fig. 8, right) is thus due to numerical dissipation in the aerodynamic calculation and not to a physical damping of the pressure perturbation.
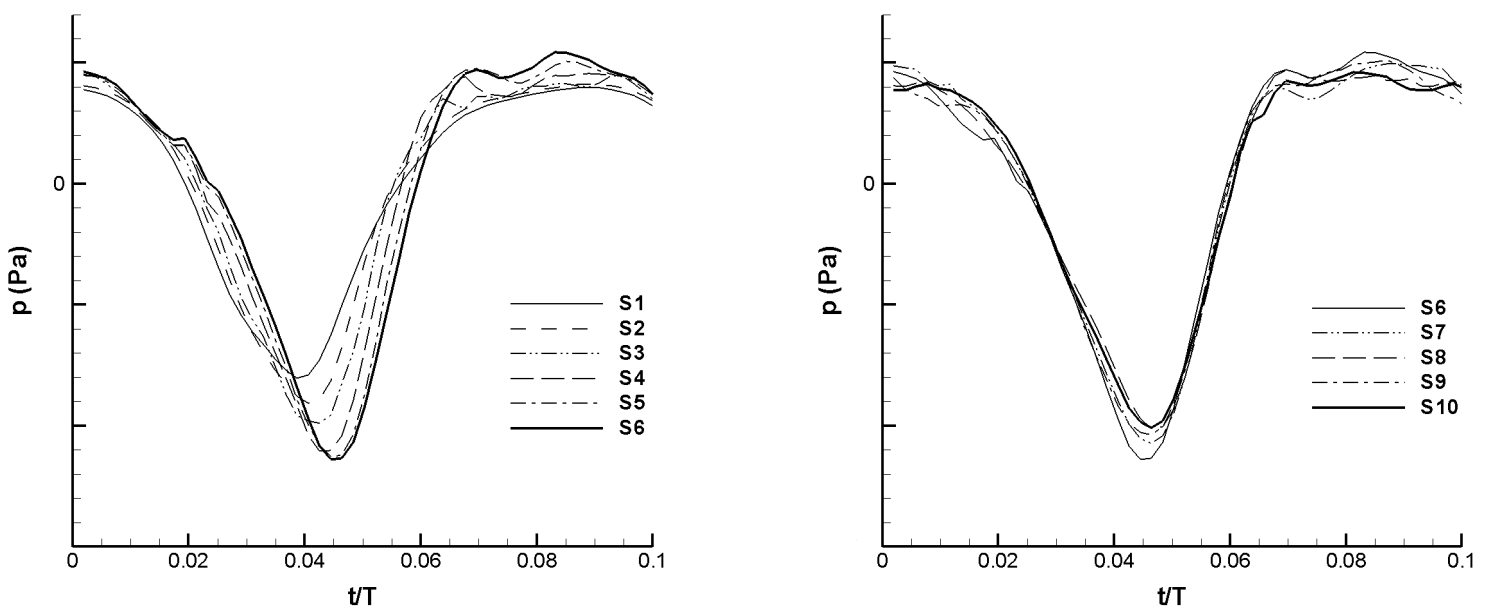

Fig. 8. Acoustic time signature in the rotor plane depending on the control surface (coarse grid). 

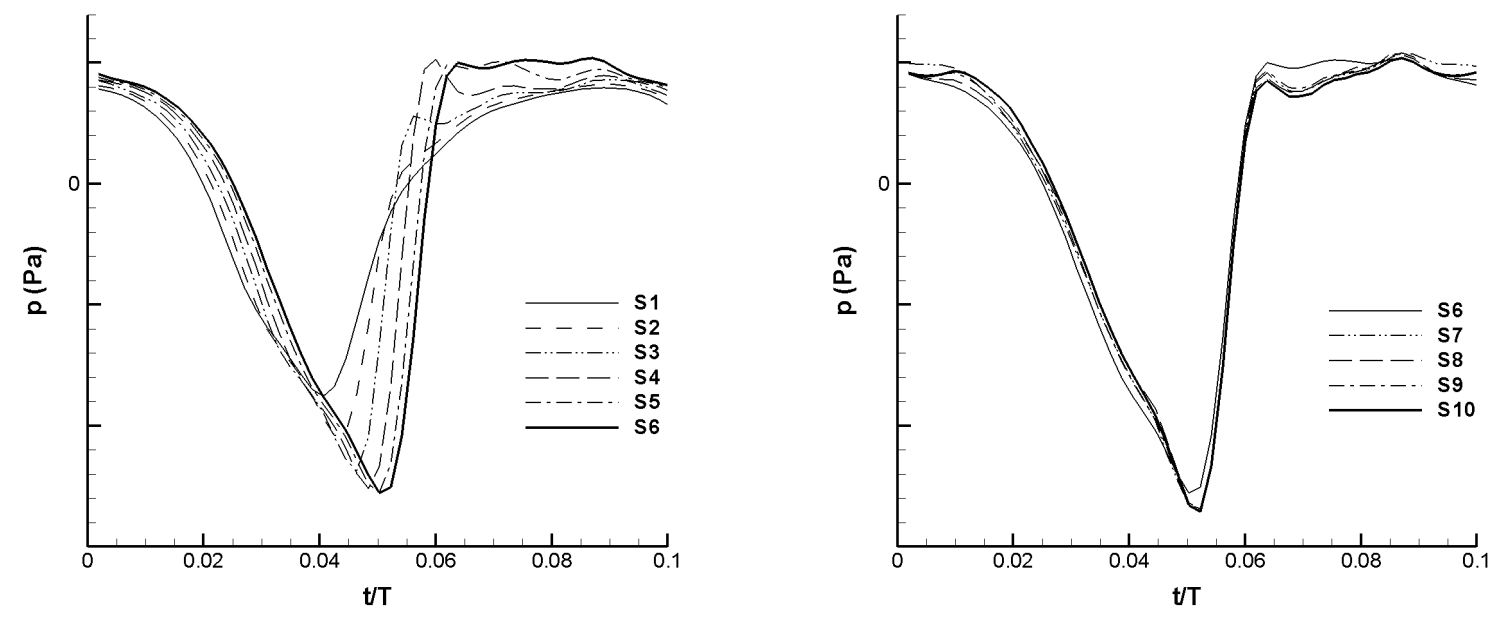

Fig. 9. Acoustic time signature in the rotor plane depending on the control surface (fine grid).

For the same observation point $(\theta=90 \mathrm{deg}$.), Fig. 10 highlights the effect of the density of the CFD grid on the predicted signal, for three particular control surfaces: $S 1$ (the closest to the blade), $S 6$ (including volume sources related to the shock) and $S 10$ (including a larger part of the blade wake). For the control surface closest to the blade (S1, Fig. 10a), the four CFD grids provide quite close results, which shows that the coarsest mesh could almost be suitable in the immediate vicinity of the blade (at least for acoustic predictions in the propeller rotation plane). For the other two control surfaces (S6 and S10), the results clearly depend on the density of the CFD grid but converge towards those found with the finest. We will see more precisely in the following section how this evolution results into the signal spectrum.

Acoustic calculations have been performed using two other time steps (T/264 and T/1034) to evaluate the sensitivity of the results to this parameter. The signal obtained does not change noticeably according to the time step (Fig. 11), which shows the robustness of the fully non-compact integration technique. A smaller time step simply tends to better describe the negative pressure peak. The deviations observed previously in Fig. 8 to Fig. 10 and those to be found with the volume method (Section 4.2) do not thus depend significantly on the acoustic time step. 
(a)

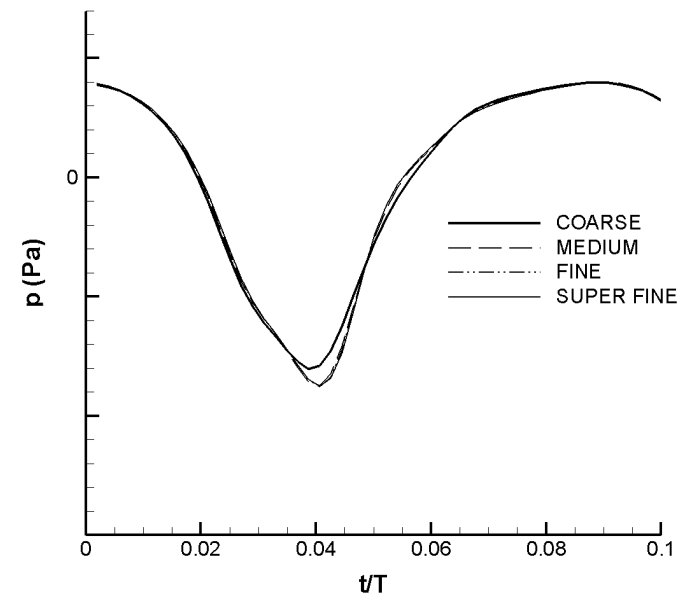

(b)

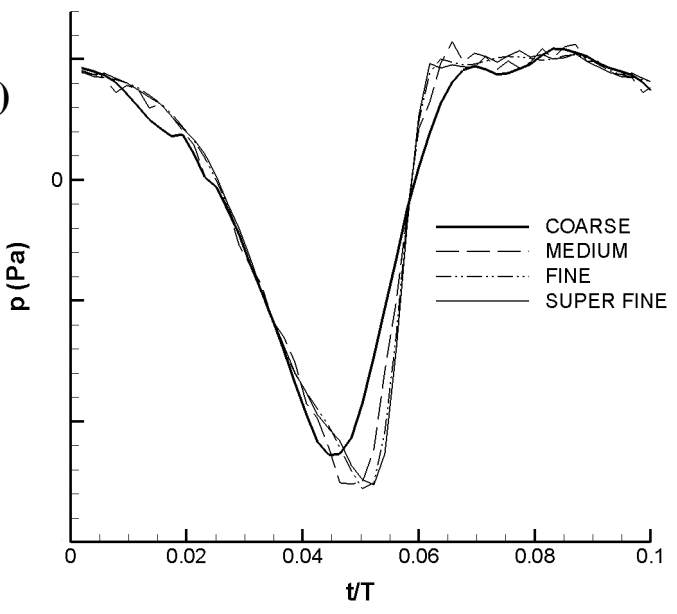

(c)

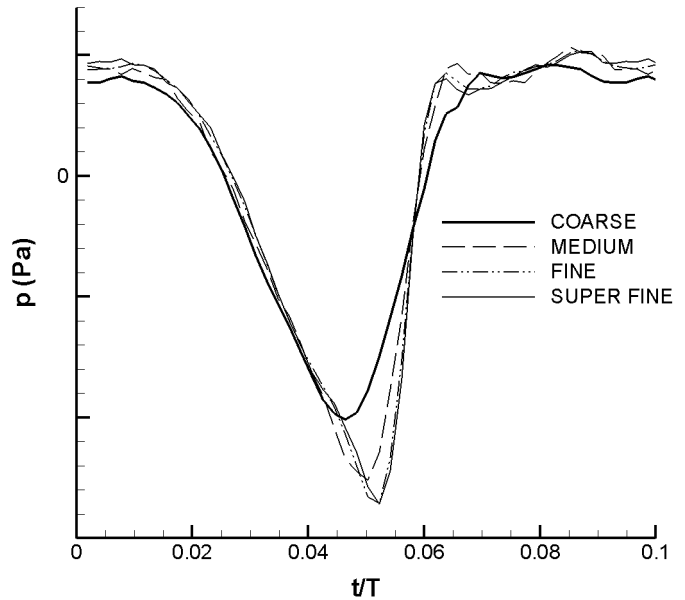

Fig. 10. Effect of the CFD grid density on the acoustic time signature in the rotor plane, for three control surfaces: (a) $S 1$, (b) $S 6$ and (c) $S 10$.

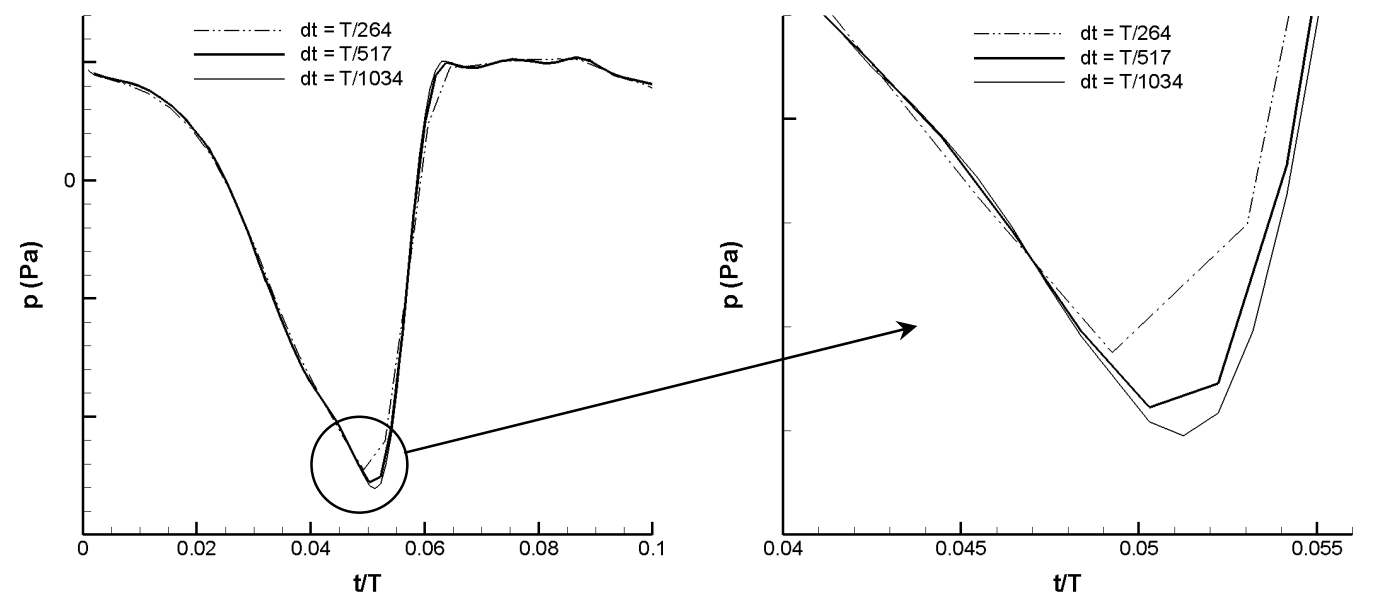

Fig. 11. Effect of the acoustic time step on the acoustic time signature in the rotor plane (control surface $S 6$, fine grid). 


\subsection{Volume calculations and comparison}

The results of the volume method applied to the volumes V6 (volume between the surfaces $S 1$ and $S 6$ ) and to the volume VA (volume between the surfaces $S 6$ and $S 10$, see Section 3.2) are plotted in figure 12 for the observation point in the rotor plane $(\theta=90 \mathrm{deg}$.), for the four CFD grids. They confirm the predominance of the volume sources related to the shock (Fig. 12, left) compared to those related to the blade wake (Fig. 12, right), as already suggested by the results of the surface method (Fig. 8 and Fig. 9). The contribution of the shock to the radiated pressure strongly depends on the grid density, but the signal converges towards that found with the finest grid (Fig. 12, left). Conversely, the contribution of the wake varies much less according to the grid density (Fig. 12, right). Therefore the numerical dissipation in the wake does not seem to produce significant volumes sources.
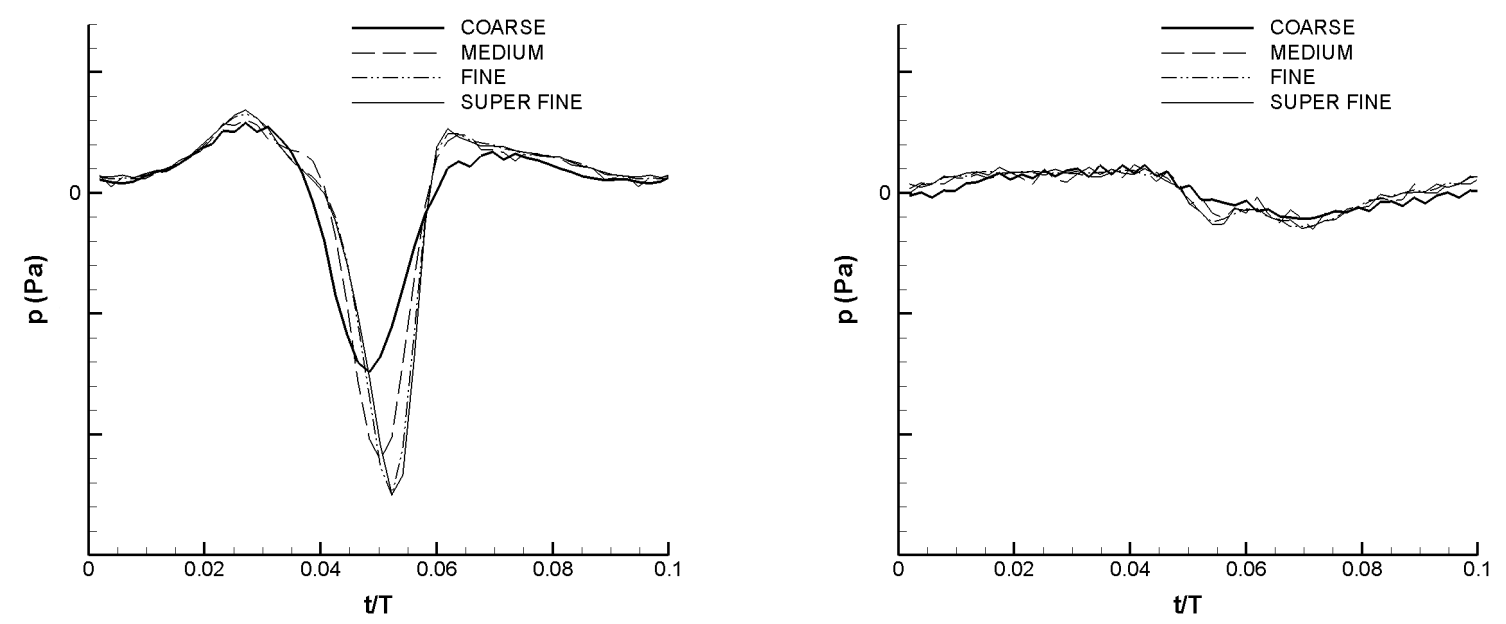

Fig. 12. Effect of the CFD grid density on the acoustic time signature in the rotor plane $(\theta=90 \mathrm{deg}$.), for volumes of integration $V 6$ (left) and $V A$ (right).

The signals obtained for the volume $V 6$ and for the volume $V 10$ (i.e. $V 6+V A$ ) are then added to those obtained for the surface $S 1$, and compared to the signals obtained respectively for the surfaces $S 6$ and $S 10$. This comparison is made in Fig. 13 for the observer point in the rotor plane $(\theta=90$ deg.). The mixed integrations on $S 1+V 6$ and on $S 1$ + V10 provide signals according to the grid density that are quite similar to those found respectively for S6 (Fig. 10b) and $S 10$ (Fig. 10c). With the coarse grid, the amplitude of the signal provided by the mixed integration on $S 1+$ $V 10$ is however slightly greater than that obtained for the surface $S 10$ (Fig. 10c). The spectral analysis that follows 
makes it possible to distinguish more precisely the differences between these various pressure signals.
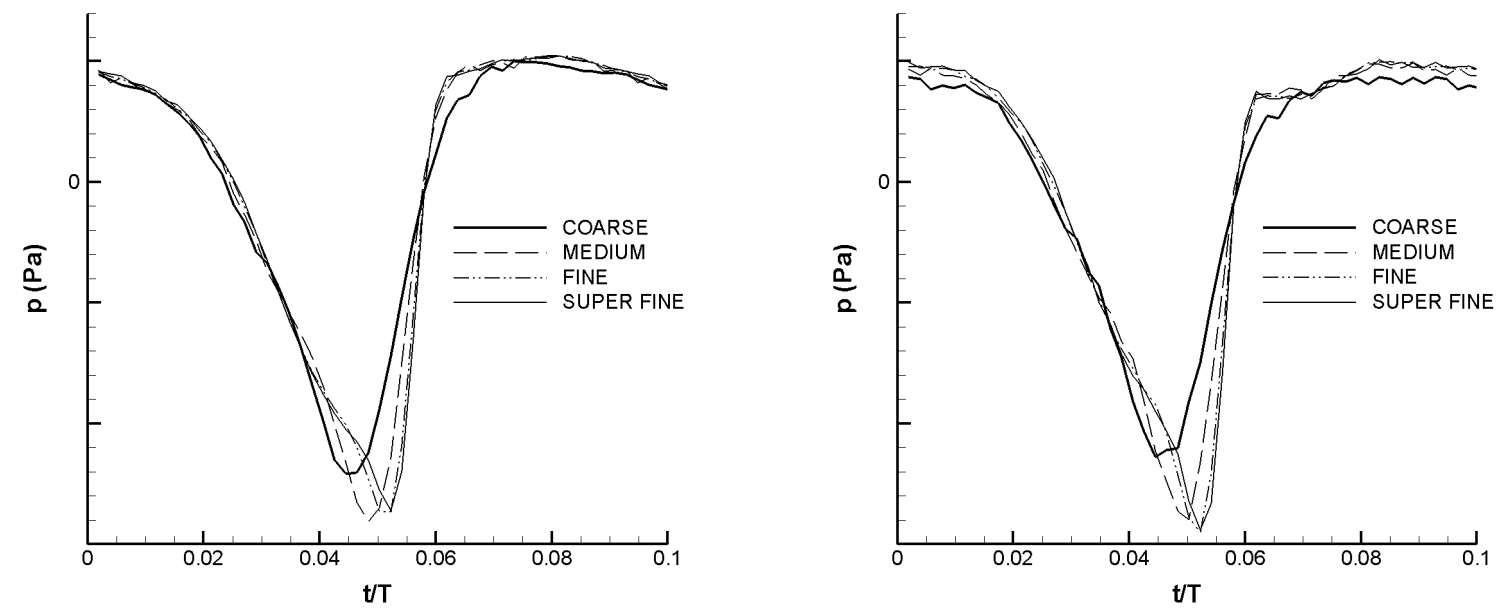

Fig. 13. Effect of the CFD grid density on the acoustic time signature in the rotor plane. (on left: integration on $S 1+V 6$, on right : integration on $S 1+V 10$ )

An example of the spectral content of the pressure radiated in the rotor plane by the sources inside the surface $S 6$ is given in Fig. 14. In this figure, the level of the first eight harmonics of the Blade Passing Frequency (BPF) is plotted for the four CFD grids and for both integration methods. The various evolutions show the effect of the grid density, which is, as expected, all the more pronounced as the frequency is high, together with a convergence of the levels with the grid density. It can be observed that the volume method leads to slightly higher levels.

\section{Remarks:}

- For the surface $S 6$ and the coarse grid, the levels have an erratic evolution beyond the fifth BPF. This defect is due to insufficient azimuthal discretization of the aerodynamic fields using this grid. Indeed, in the present case of rotating stationary fields, each BPF corresponds to an azimuthal mode of the aerodynamic perturbations. Therefore the calculated levels are reliable only for the BPF corresponding to azimuthal modes correctly described by the grid for FW-H calculations. In this study, levels corresponding to modes represented by less than eight points have not been considered as reliable and are not plotted thereafter. For the first eight harmonics studied here, in practice, this criterion applies only to the coarse grid (see Table 2).

- The various level evolutions can be related to the capability of the CFD computation to propagate a perturbation according to the grid density. In the present case of a second-order accurate in space scheme, it 
is generally considered that twenty cells per wavelength are necessary for a correct calculation of the acoustic propagation up to the integration surface. An accurate calculation of the cut-off frequency of the CFD calculation is possible only for a Cartesian grid with constant step, which is not the case in this study carried out with cylindrical grids. However, a rough estimate of this frequency can be made from the average cell size in the region beyond the sonic cylinder, which is a key region for the BPFs amplitudes. This average value is calculated in the azimuthal direction, because, as mentioned above, the acoustic radiation is directly related to the azimuthal modes. The cut-off frequencies obtained for each grid are indicated in Table 5. The corresponding cut-off BPFs are relatively consistent with those deduced from the evolutions found with the surface method, which relies on the transport of disturbances up to the surface $S 6$ (Figure 14, left). These cutoff BPFs are less easy to determine starting from the results of the volume method, the various curves separating more gradually (Figure 14, right).

\section{Table 5}

Estimation of the cut-off frequency of the CFD calculations, depending on the grid

\begin{tabular}{ccccc}
\hline Grid & Coarse & Medium & Fine & Super fine \\
Average cell size in the supersonic area (m) & 0.0348 & 0.0239 & 0.0160 & 0.0137 \\
Cut-off frequency (Hz) & 427 & 621 & 928 & 1084 \\
Corresponding cut-off BPF & 2.9 & 4.3 & 6.4 & 7.4 \\
\hline
\end{tabular}
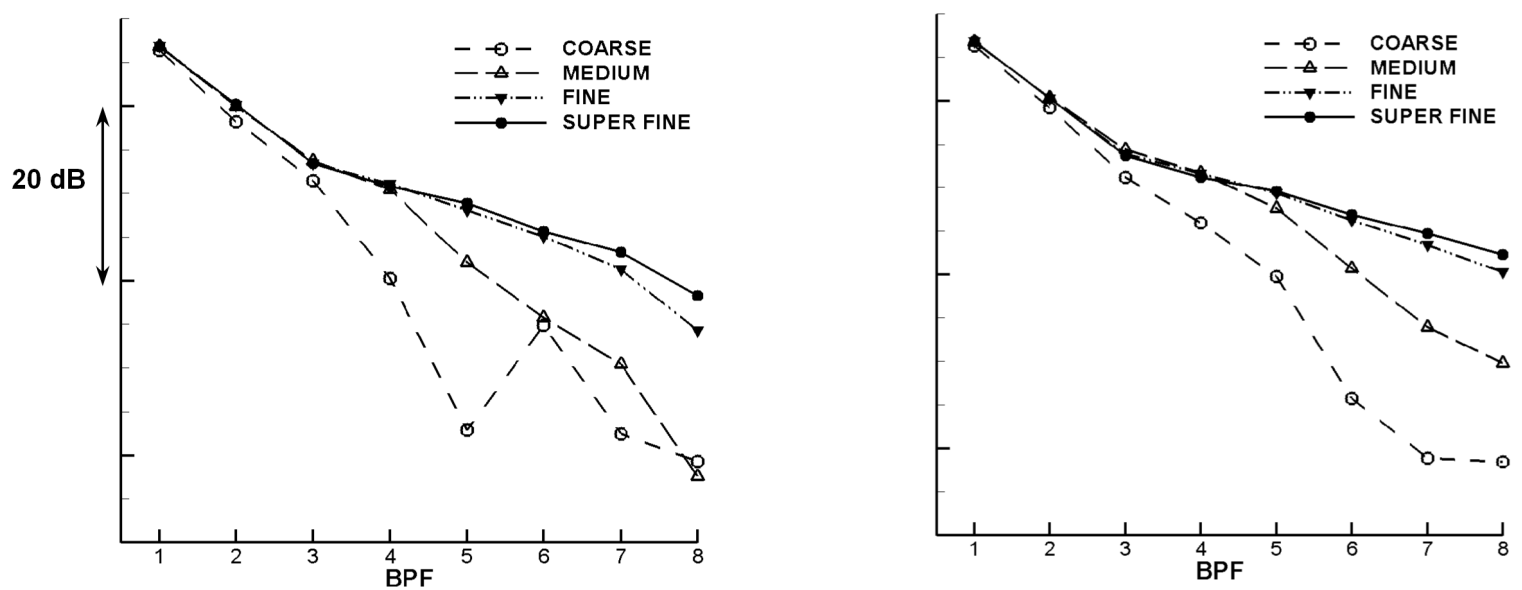

Fig. 14. Examples of the effect of the CFD grid density on the spectra of the pressure radiated in the rotor plane (on left: integration on $S 6$, on right: integration on $S 1+V 6$ ). 
A more complete view of the evolution of the acoustic radiation depending on the source volume considered, is given in Fig. 15. In this figure, the maximum of the OverAll Sound Pressure Levels (OASPL) and the maxima of the Sound Pressure Levels (SPL) of the first eight BPFs on the semi-circle of observation around the rotor are plotted for both integration methods, as a function of the integration surface $\mathrm{Si}$ or the integration volume $V i$, for three of the four CFD grids.

The results obtained with the fine grid show, from the spectral point of view, the construction of the signal as more volume sources are taken into account in the acoustic calculation (Fig. 15c, Index 1 to 6). These volume sources result from a nonlinear acoustic propagation in the near vicinity of the propeller, due to the high amplitude of the initial disturbance, and lead to a stiffening of the pressure perturbation (see Fig. 9 left). This stiffening results in a fairly fast growth of the high harmonics (Fig. 15c, Index 1 to 4). Then, the level of the highest harmonics tends to decrease, indicating a slight damping of the signal (Index 4 to 6). This signal damping can be attributed to numerical dissipation in the aerodynamic calculation, since it mainly affects the BPFs 6 to 8 , in relatively good agreement with the cut-off frequency of the fine grid (see Table 5 and Fig. 14) and increases with the medium grid (Fig. 15b). The volume method and the surface method give similar evolutions but with slightly higher levels with the former. One can wonder what precisely causes the deviations between the two methods while they are theoretically equivalent. These deviations could be due to the fact that the physical viscosity of the fluid is taken into account in aerodynamic calculations (RANS calculations) but not in acoustic calculations (viscous source term neglected in both acoustic methods, see Section 2.1). To illustrate it, one can take the example of a flow with viscous effects confined in a limited volume. The surface method applied to the boundary of this volume will provide the same results with or without the viscous source term (viscous source term equal to zero on the integration surface). In contrast, the volume method applied to this volume will provide these results only if the viscous source term is taken into account. Therefore, the results of the two methods without the viscous source terms will be different.

However, in the present case, the viscosity of the fluid is not a cause of significant deviations in the acoustic results. Indeed, this physical viscosity does not depend on the grid density, whereas the deviations tend towards zero when the grid is refined, as will be confirmed thereafter by the directivity plots (Fig. 18).

On the other hand, the artificial viscosity necessary to the stability of the CFD calculation (coefficients k2 and k4 of the cell-centered Jameson scheme) can be the cause of the deviations. This numerical viscosity limits the gradients in the whole volume of fluid, and consequently the amplitude of the high harmonics with both methods. It increases 
as the grid is coarser, and the level of these harmonics decreases accordingly (Fig. 15b and Fig. 15a). Its effect may be slightly weaker with the volume method (which adds the contribution of each elementary volume source) than with the surface method (which works starting from the state of the aerodynamic fields on the integration surface). The numerical viscosity also tends to damp the pressure perturbation throughout its propagation in the radial direction and leads to decreasing levels for the indexes 4 to 6 , with both methods. For the volume method, this level decay means that the numerical dissipation creates volume sources which reduce the levels of the high harmonics by phase-shifted contributions. An example of these phase-shifted contributions is shown in Fig. 16 for the BPF6 and for the volume of integration $V 5-V 4$, for the fine grid. These volume sources eventually have the same effect on the radiated pressure as that of damped perturbations on the integration surfaces.
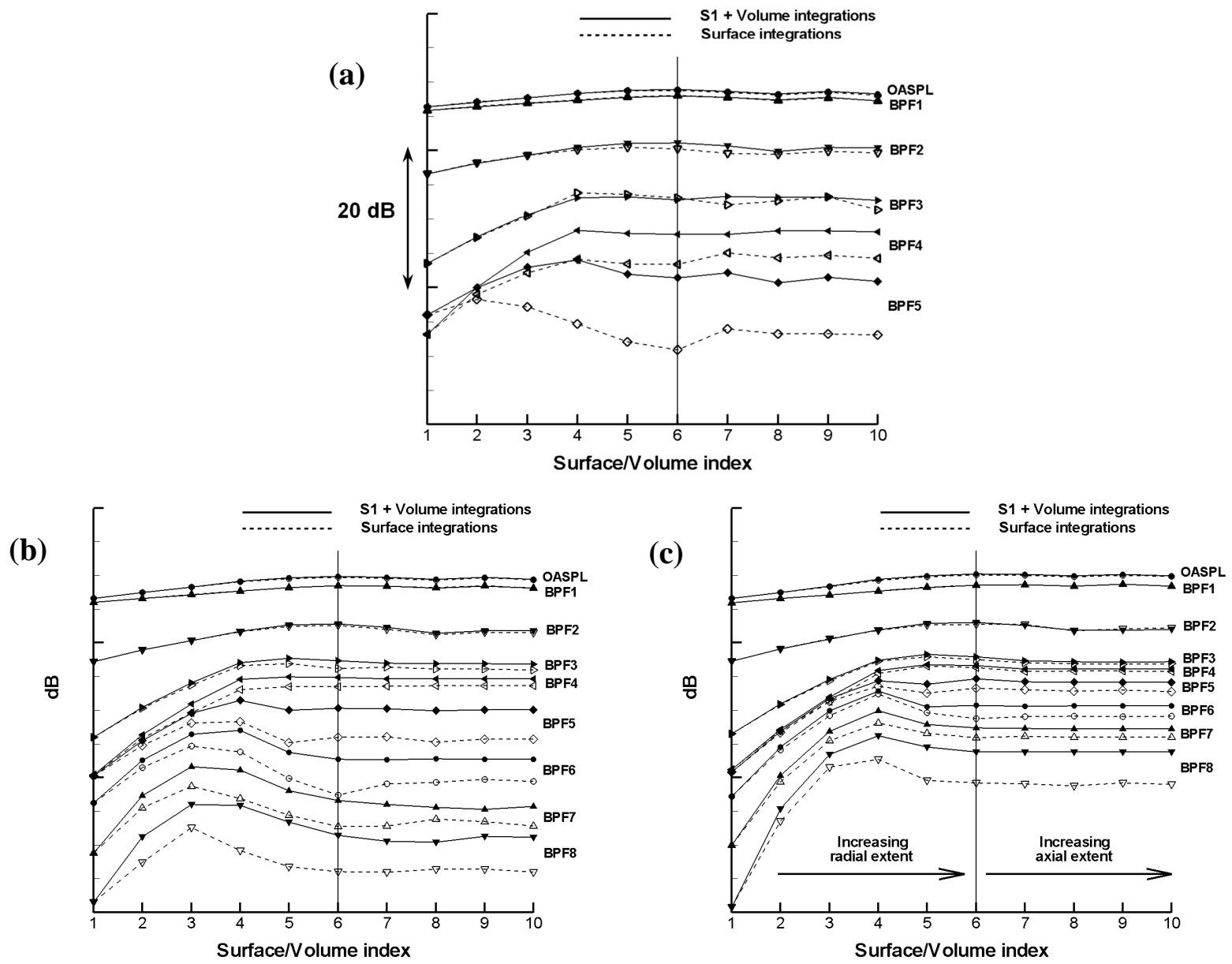

Fig. 15. Maximum OASPL and maximum SPL of the BPF harmonics, as a function of the source volume considered, for (a) the coarse grid, (b) the medium grid and (c) the fine grid. 


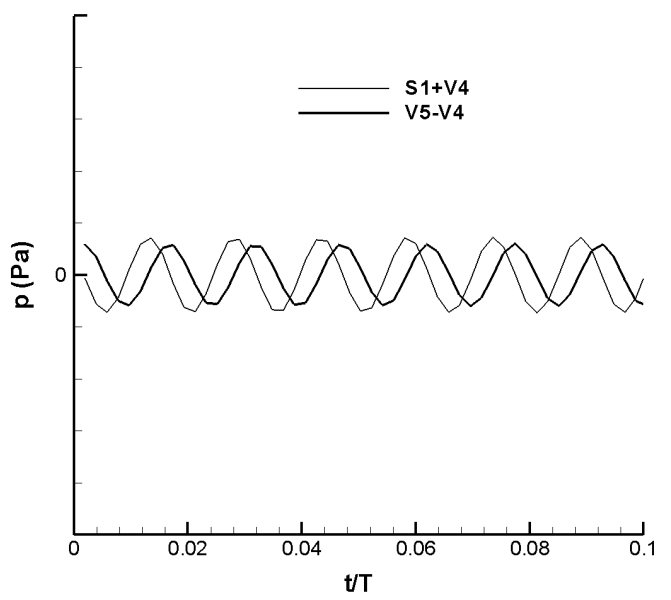

Fig. 16. (S1+V4) and (V5-V4) contributions to BPF6 (fine grid, observer in the rotor plane).

Remark: With CFD methods or resolution schemes other than those used in this study, the artificial viscosity required for the stability of the aerodynamic calculation is introduced by other means than the coefficients $\mathrm{k} 2$ and $\mathrm{k} 4$ mentioned above, but its effects on the acoustic calculations can be expected to be similar to those observed here for both integral methods.

Additional comments can be made regarding the coarsest grid (Fig. 15a). The results obtained with this grid illustrate the difficulties that can be encountered with the surface method when the dissipation in the CFD calculation is too high in the region of the acoustic sources. Depending on the integration surface used, either it does not enclose enough sources (here, surfaces $S 1$ to $S 3$ for the BPF1 to the BPF4), or the numerical dissipation affects the aerodynamic fields too strongly (here, surfaces $S 3$ to $S 6$ for the BPF5). With this CFD grid, the volume method seems to be much less sensitive to the numerical dissipation, especially for the fifth BPF. However, the predicted level for this frequency remains $13 \mathrm{~dB}$ lower than that obtained with the fine grid (Fig. 15c).

A synthetic view of the benefit of the volume method is given in Fig. 17, in which the maximum levels obtained by both integration methods are plotted as a function of the BPF, respectively for the surface $S 10$ and the volume $V 10$, for the four CFD grids. This figure confirms that the volume method tends to improve the results. However, as discussed above, it palliates only very partially the lack of precision of the aerodynamic fields provided by insufficiently fine grids. This figure also shows the need of very fine CFD grids to correctly predict the high frequencies produced by shocks in transonic flows. 


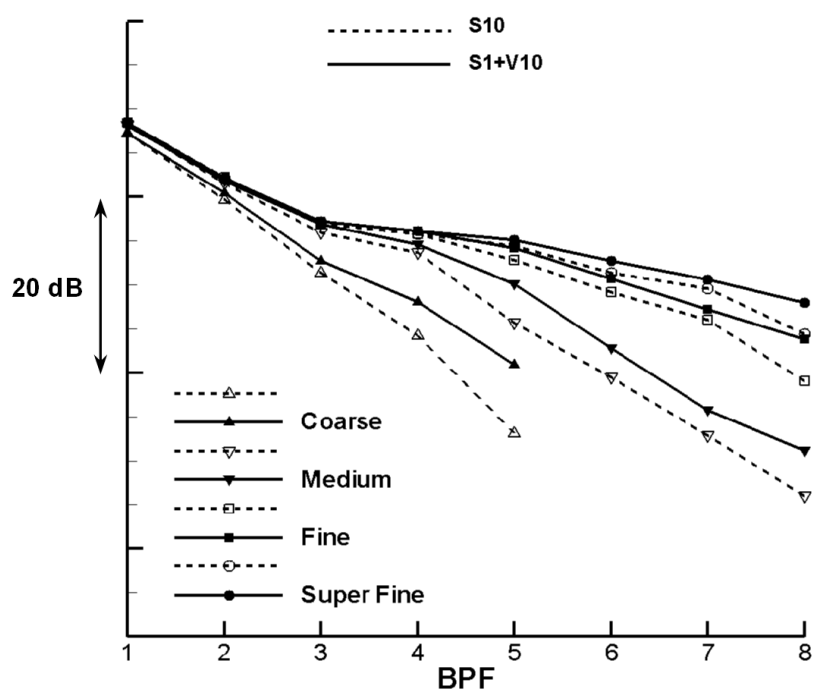

Fig. 17. Effect of the CFD grid density on the maximum SPL of the BPF harmonics, for both surface and volume methods.

In addition and to confirm the previous analysis for other observer points, the directivity of BPF 1,3 and 5 are plotted for the four CFD grids and for both integration methods (Fig. 18).

With the super fine grid (Fig. 18d), the numerical dissipation is very weak and the directivities provided by both integration methods are practically identical. With the other three grids, the differences between the results of the surface and volume methods gradually increase as the CFD grid is coarsened (except for the BPF1, of which the level does not depend on the grid nor the integration method). The volume method leads to generally slightly higher levels and especially to less chaotic directivities than those provided by the surface method.

In conclusion, the volume method provides slightly better results than the surface method in case of CFD grids that are not fine enough for accurate acoustic predictions, however the gain seems low compared to the computation cost of the volume integration. 

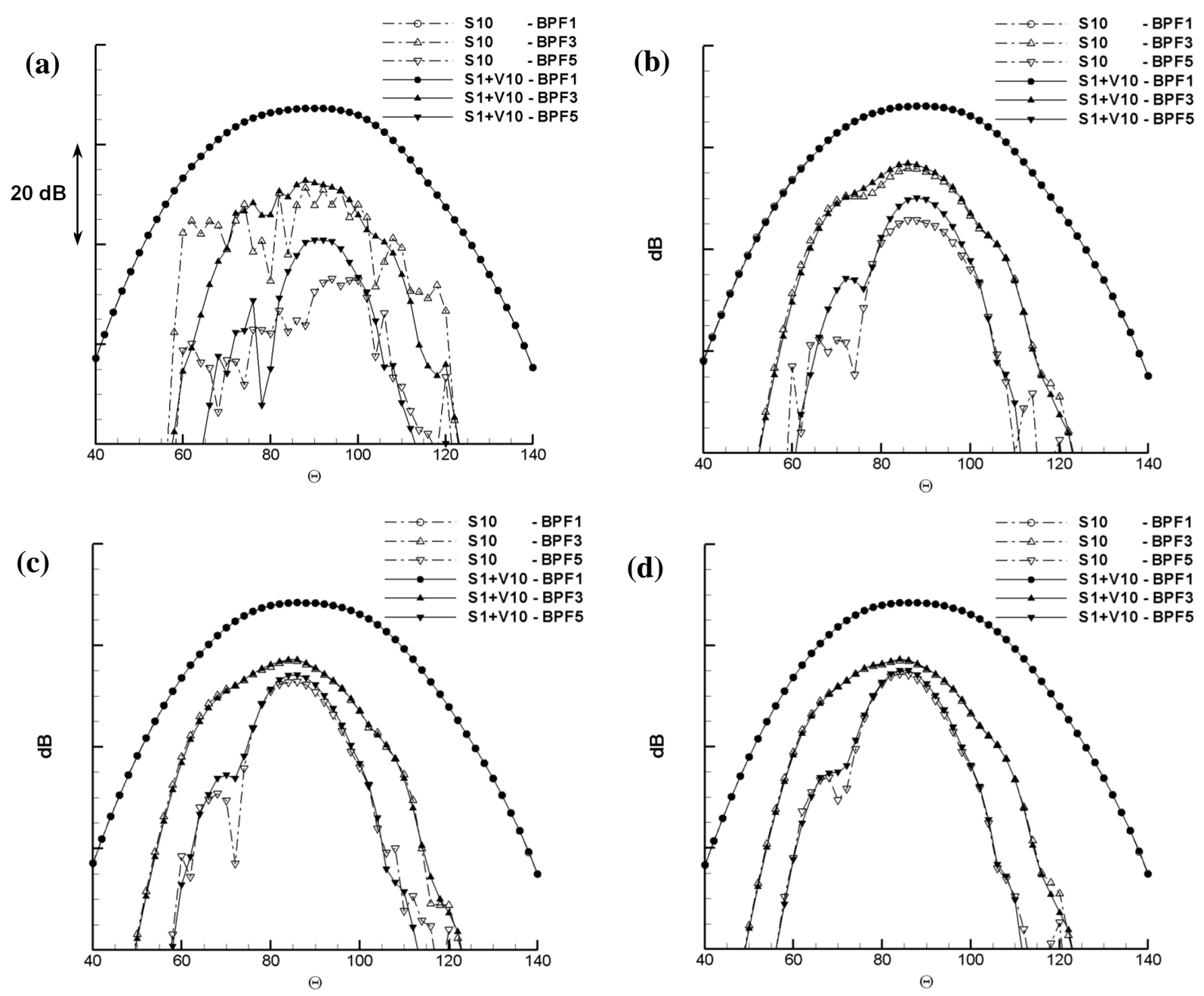

Fig. 18. Directivities predicted by surface and volume methods, for (a) the coarse grid, (b) the medium grid, (c) the fine grid and (d) the super fine grid.

\subsection{Spatial distribution of the noise sources}

In this section, two techniques for the analysis of volume sources are explored. The main objective is to determine which regions of the flow contribute the most to acoustic radiation. This information, that the surface method cannot provide, can be used to optimize the CFD grid, for example by showing the regions where the mesh must be refined.

The first technique, rather conventional, consists in calculating the local value of so-called 'quadrupole' term $\partial^{2} T_{i j} / \partial Y_{i} \partial Y_{j}$. This double divergence of the Lighthill tensor constitutes the source term of the volume integral when the second spatial derivative in the expression (1) is passed inside the volume integral (see [10], for example). 
This source term may thus be able to serve the intented purpose.

Since the viscous term $e_{i j}$ is neglected in the calculations (see section 2.1), the quadrupole terms are here reduced to the shear term $\partial^{2}\left(\rho u_{i} u_{j}\right) / \partial Y_{i} \partial Y_{j}$ and to the "entropy", term $\partial^{2}\left(p^{\prime}-a_{0}^{2} \rho^{\prime}\right) / \partial Y_{i} \partial Y_{i}$. The contribution of each of these two terms to the signal radiated in the rotor plane $(\theta=90 \mathrm{deg}$. $)$ is plotted in Fig. 19. This figure shows that, for this propeller in transonic operating conditions, the contribution of the volume sources is mainly produced by the shear term.

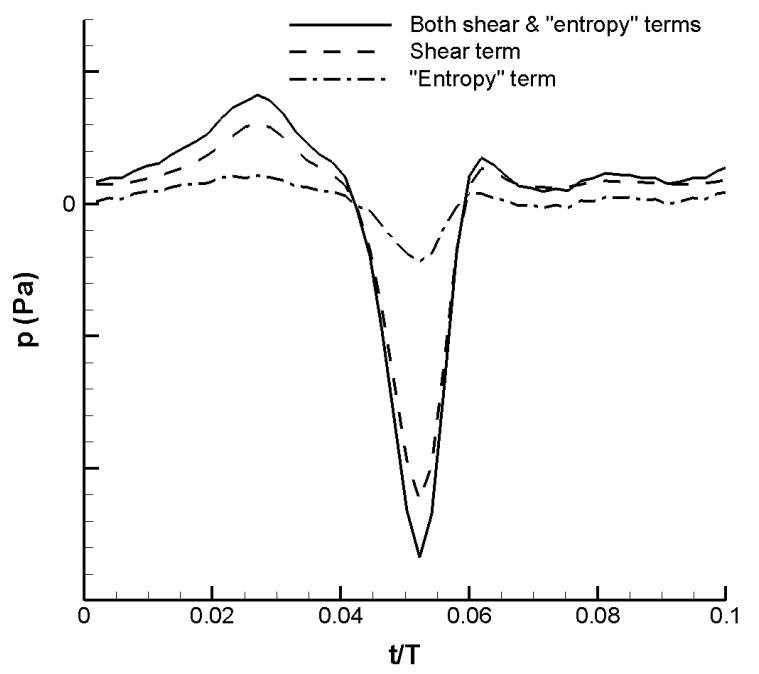

Fig. 19. Contributions of the shear term and of the "entropy" term to the pressure radiated in the rotor plane (integration volume V10, fine grid).

The isosurfaces of these two terms confirm that the shear term is dominant in the shocks, however the values of the isosurfaces must be chosen carefully to highlight it (Fig. 20). Indeed, the source terms are much weaker there $(\mid$ level $\mid<50)$ than in the wake and the blade tip vortex $((\mid$ level $\mid>1000$ in the vortex core), which yet are not significant noise sources compared to the shocks. Despite their magnitude, the sources (in the sense of Lighthill) in the wake and the blade tip vortex have, after spatial integration, an overall contribution in fact negligible, because they are arranged on successive layers of opposite signs, and their radiations cancel each other.

These iso-surfaces confirm, for this propeller, the well-known fact that the quadrupole term is not a quantity that determines the actual noise sources easily. In practice, it is preferable to plot a particular component of the

${ }^{2}$ At the first order, $\left(p^{\prime}-a_{0}^{2} \rho^{\prime}\right)=\frac{p_{0}}{c_{v}}\left(S-S_{0}\right)$ where $\left(S-S_{0}\right)$ is the entropy variation. 
quadrupole term that can locate the phenomenon concerned better than the shear or entropy original terms would do.

For example, in the case of this propeller, the simplified shear term $\partial^{2}\left(\rho u_{1} u_{1}\right) / \partial Y_{1}^{2}$ (Fig. 21, left) and especially the pressure term $\Delta p$ (Fig. 21, right) seem more effective in distinguishing shock-related noise sources from other sources (much higher relative levels in the shocks, compared to Fig. 20).
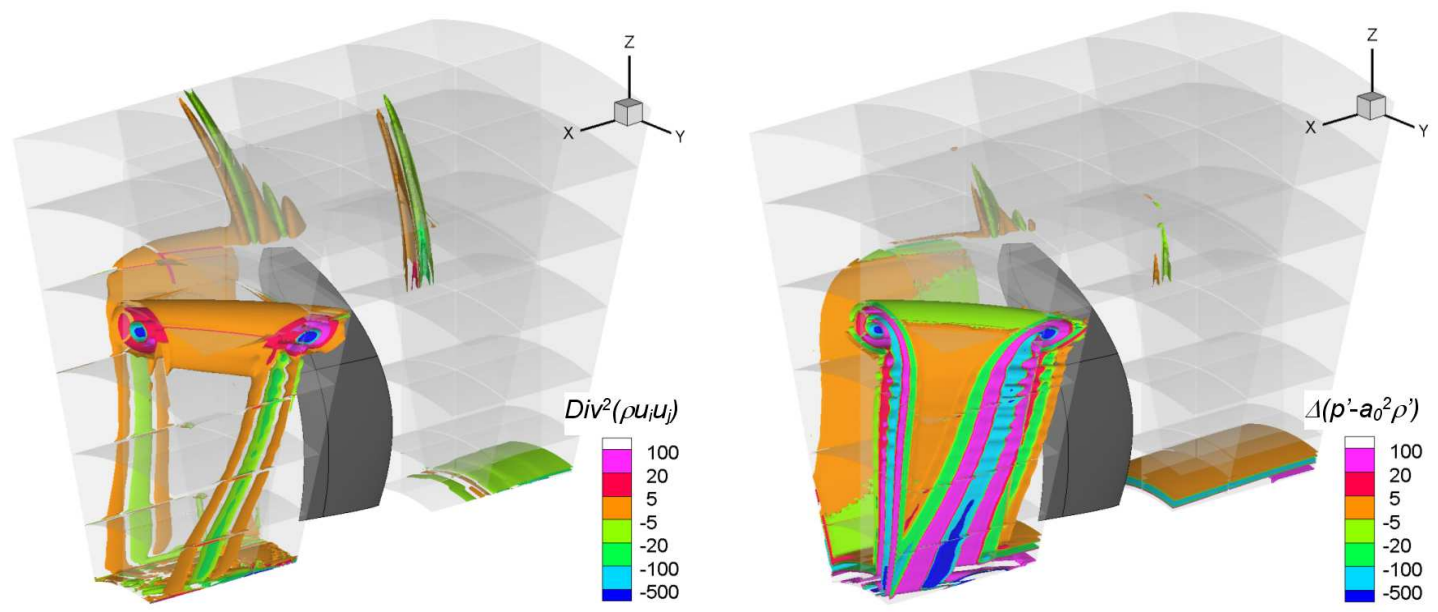

Fig. 20. Isosurfaces of the normalized shear (on left) and "entropy” (on right) quadrupole terms - Fine grid.
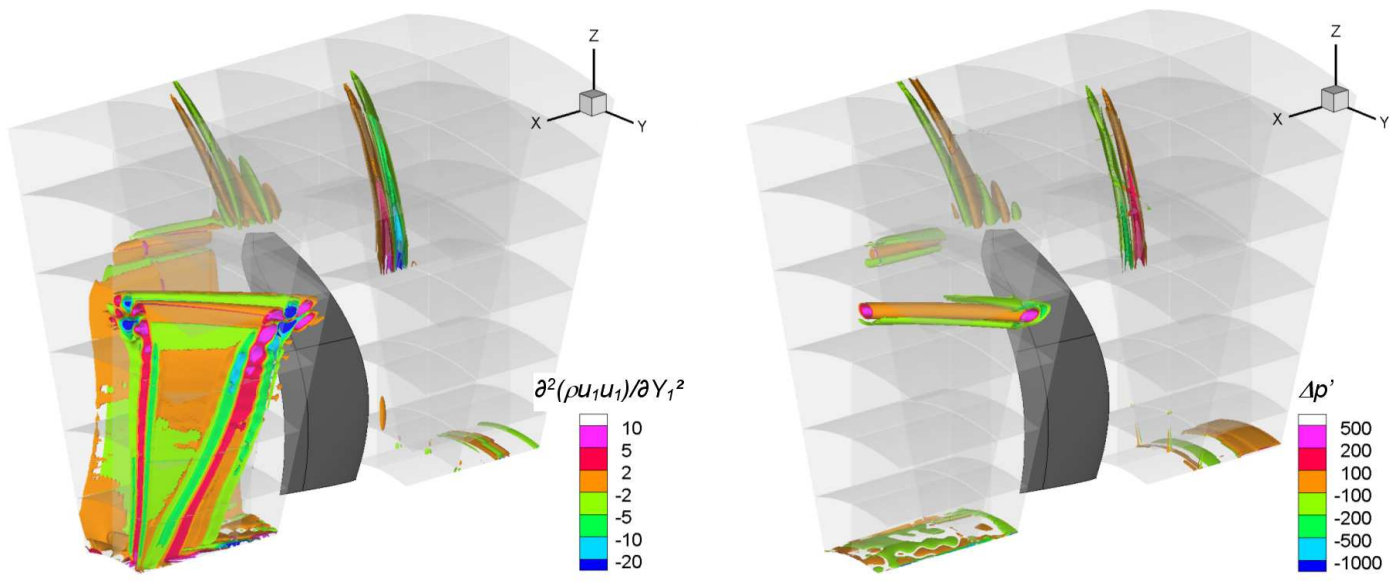

Fig. 21. Isosurfaces of simplified shear (on left) and pressure (on right) terms - Fine grid. 
The second technique for the analysis of the volume sources consists in determining the elementary acoustic time signature radiated by each cell of the grid, for a given point of observation (in $\mathrm{Pa} / \mathrm{m}^{2}$ and in $\mathrm{Pa} / \mathrm{m}^{3}$ respectively for the surface method and for the volume method). This technique has the drawback of providing a result that depends on the observation point, but the advantage of providing the precise contribution of each cell to the sound radiation, and not only the magnitude of a local source term. It has been applied to the surface of the blades of open rotors, with an analysis of the amplitude and the phase of the radiated pressure, for various BPFs [31][32]. For reasons of memory cost with the volume method, this analysis is limited here to the root mean square (rms) value.

The local contributions to the noise radiated in the rotor plane ( $\theta=90 \mathrm{deg}$.$) are plotted separately for the shear$ term and for the entropy term in Fig. 22. For this transonic propeller, the rms value of the shear term local contribution appears to be a good indicator of the noise sources (Fig. 22, left). In particular, the wake and the blade tip vortex do not appear as noticeable noise sources.

In contrast, the rms value of the "entropy" term local contribution is unusable (Fig. 22, right). In the vicinity and beyond the sonic cylinder, the levels are relatively uniform over large regions and completely mask the shocks. This is due to the difference between the average values over a blade revolution and the values at infinity, for pressure and density. This difference is produced by the thrust of the propeller and is seen as a constant source that rotates with the cell. In the vicinity and beyond the sonic cylinder, the transonic kinematics of the cells considerably amplifies this spurious component which, in fact, only distorts the level at the blade passage frequency (BPF1) and the rms value of the local contribution. An analysis of several BPFs, as it has been done for the surface method [31][32], would not have encountered this problem. Calculations performed starting from pressure and density fields from which the average value over a blade revolution has been subtracted, confirm this explanation and a weak contribution of the "entropy" term compared to the shear term (Fig. 23). 


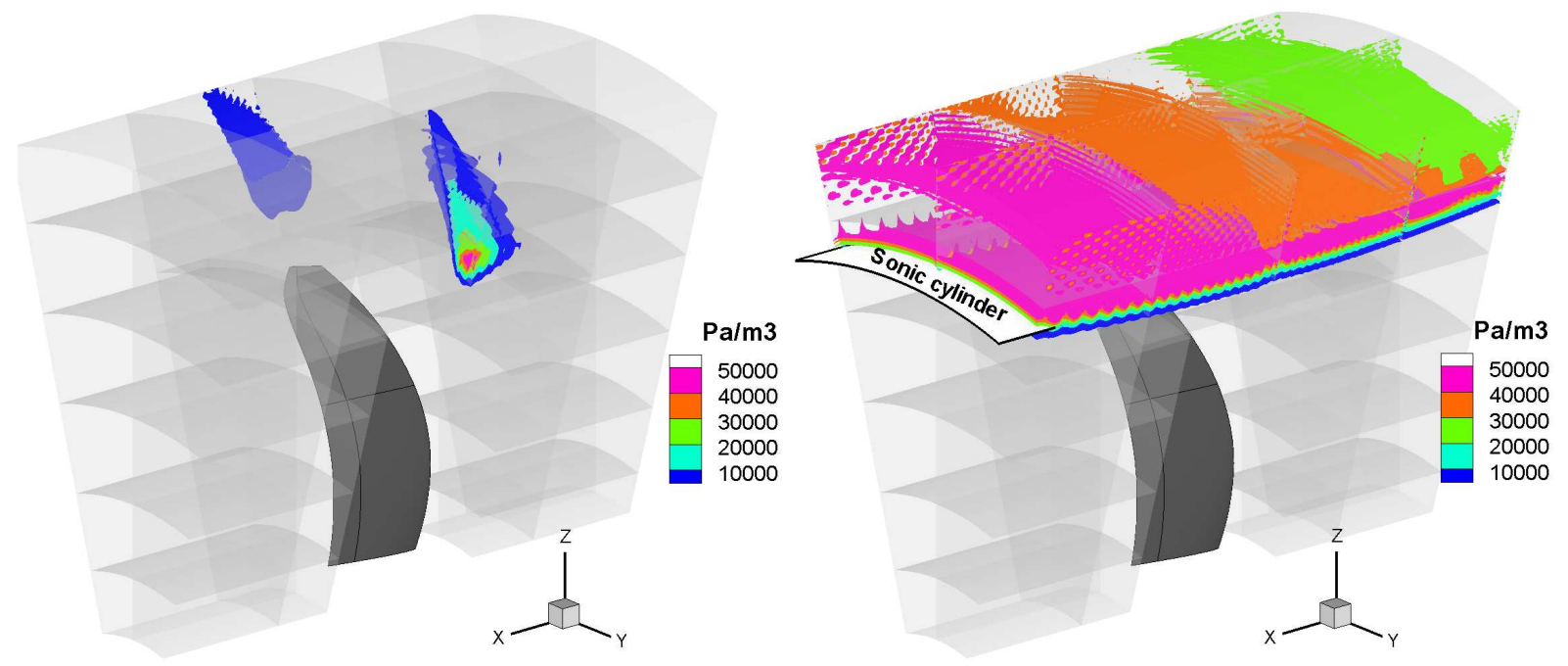

Fig. 22. Isosurfaces of the shear term (on left) and of the "entropy" term (on right) local contributions to the pressure radiated in the rotor plane - Fine grid.

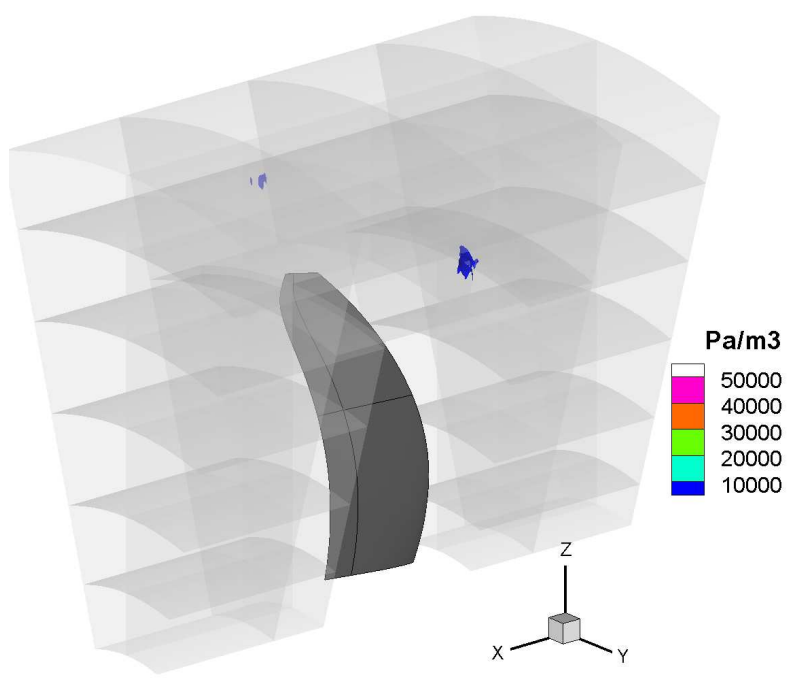

Fig. 23. Isosurfaces of the "entropy" term local contribution to the pressure radiated in the rotor plane, starting from modified pressure and density fields - Fine grid. 


\section{Conclusion}

Acoustic calculations using two different approaches of the FW-H integral formulation were conducted for a transonic propeller. The first indirectly takes into account the volume sources created by the propeller, by means of an integration on a closed surface encompassing these sources. The second takes them directly into account by means of a volume integration without any simplification that could distort the comparison. For both approaches, the calculations were greatly facilitated by a specific integration technique. Thanks to this fully non-compact integration technique, acoustic calculations were performed directly from data provided in the rotating frame, i.e. with supersonically rotating emission points. No projection of the aerodynamic fields on a fixed refined grid, which would have considerably increased the cost of the acoustic calculations and would have been a potential source of inaccuracies, was needed.

The surface and volume calculations were carried out for four CFD meshes of different densities, and thus for four different levels of numerical dissipation in aerodynamic calculation, in order to evaluate the sensitivity of both methods to this numerical dissipation. Both integral methods converge to the same result as the CFD mesh is refined, which contributes to validate the aerodynamic calculations and confirms the theoretical equivalence of the two acoustic methods. The results show the effect of the artificial viscosity which is introduced in the CFD calculation for its stability, on acoustic predictions. This artificial viscosity, which limits the gradients and damps the disturbances, leads to an underestimation of the acoustic radiation, all the more pronounced as the frequency is high. This damping of the high frequencies is quite similar with both integration methods because the artificial viscosity has comparable effects in both of them. On the one hand, the gradients limitation during the construction phase of the acoustic signal leads to an underestimation of the source terms in both methods. On the other hand, the damping of the pressure perturbation during its propagation results in lower levels on the control surface, for the surface method, and in volume sources that reduce the radiated pressure by a phase effect, for the volume method. This numerical dissipation has slightly smaller consequences with the volume integration, but the improvement brought seems weak compared to its computation cost.

In addition, two techniques for determining the regions of the dominant acoustic sources were explored. The first one, rather conventional, consists in calculating the local value of the double divergence of the Lighthill tensor (the 'quadrupole term'). The second, less known, consists in calculating the elementary acoustic time signature radiated by each cell of the grid towards a given point of observation. Calculations made with the first technique confirm that 
the quadrupole term is not a quantity that determines the actual noise sources easily, because the levels there may be low compared to regions that contribute little or not at all to the sound radiation. They also showed that specific terms chosen according to the phenomenon concerned may be better indicators of the real noise sources than the original shear and entropy terms. The second method seems more effective in determining the local contributions to the far-field radiated noise. In the present case of a flow accelerated by the propeller thrust, the rms value of the elementary acoustic time signature is however unusable if it includes the contribution of the entropy term, because of the predominance of a spurious component at the propeller blade passage frequency in this term. A spectral analysis of the elementary acoustic time signatures makes it possible to overcome this problem but can be costly in terms of data storage with the volume method.

In conclusion, in view of its computational cost (about two orders of magnitude greater than the surface method) and of the weak improvement brought to the acoustic predictions, the volume method does not seem very attractive for acoustic predictions of transonic propellers. It can nevertheless be a complementary tool for in-depth analyses of the aerodynamic fields or to consolidate the results of the surface method, for instance. It would also be interesting to reconsider the issue for acoustic predictions of turbulent flows such as jets.

\section{Acknowledgements}

The author gratefully acknowledges A. Giauque, B. Ortun and B. Rodriguez for providing the CFD input data of this acoustic study.

Special thanks to Julie for her invaluable help. 


\section{References}

[1] Ffowcs Williams J. E., Hawkings D. L, Sound Generation by Turbulence and Surfaces in Arbitrary Motion, Philosophical Transactions of the Royal Society of London 1969;264(A1151):321-342.

[2] Lighthill M. J., On Sound Generated Aerodynamically, 1: General Theory, Proceedings of the Royal Society of London 1952;A221:564-587.

[3] Di Francesantonio P., A new boundary integral formulation for prediction of sound radiation, J. Sound Vib. 1997;202(4):491-509.

[4] Blacodon D., Elias G., Level Estimation of Extended Acoustic Sources Using a Parametric Method, Journal of Aircraft 2004;41(6).

[5] Deneuve A., Druault Ph., Marchiano R., Sagaut P., A coupled time-reversal/complex differentiation method for aeroacoustic sensivity analysis: towards a source detection procedure, J. Fluid Mech., 2010;642:181-212.

[6] Brentner K. S., An efficient and robust method for predicting helicopter rotor high-speed impulsive noise, $34^{\text {th }}$ AIAA Aerospace Sciences Meeting and Exibit, Reno, NV, January 1996.

[7] Farassat F., Brentner K. S., Supersonic quadrupole noise theory for high-speed helicopter rotors, Journal of Sound and Vibration 1998;218(3):481-500.

[8] Ianniello S., Quadrupole Noise Predictions Through the Ffowcs Williams-Hawkings Equation, AIAA Journal 1999;37(9):1048-1054.

[9] Rahier G., Prieur J., An efficient Kirchhoff integration method for rotor noise prediction starting indifferently from subsonically or supersonically rotating meshes, AHS forum 53, Virginia Beach, May 1997.

[10] Prieur J., Rahier G., Aeroacoustic integral methods, formulation and efficient numerical implementation, Aerospace Science and Technology 2001;5(7):457-468.

[11] Brentner K. S., Farassat F., Analytical comparison of the acoustic analogy and Kirchhoff formulation for moving surfaces, AIAA Journal 1998;36(8):1379-1386.

[12] Rahier G., Huet M., Prieur J., Additional terms for the use of Ffowcs Williams and Hawkings surface integrals in turbulent flows, Computers and Fluids 2015;120:158-172.

[13] Delrieux Y., Prieur J., Rahier G., Drousie G., A new implementation of aeroacoustic integral method for supersonic deformable control surfaces, $9^{\text {th }}$ AIAA/CEAS Aeroacoustics Conference and Exhibit, Hilton Head, May 2003.

[14] Purcell T. W., CFD and Transonic Helicopter Sound, $14^{\text {th }}$ European Rotorcraft Forum, Milan, Italy, September 1988.

[15] Prieur J., Costes M., Baeder J. D., Aerodynamic and Acoustic Calculations of Transonic Non-Lifting Hovering Rotors, Journal of the American Helicopter Society 1996;41(2):17-26. 
[16] Baeder J. D., Euler Solutions to Non Linear Acoustics of Non-Lifting Hovering Rotors, $16^{\text {th }}$ European Rotorcraft Forum, Glasgow, Scotland, September 1990.

[17] Prieur J., Rahier G., Comparison of Ffowcs Williams-Hawkings and Kirchhoff rotor noise calculations, AIAA Paper 98-2376, $4^{\text {th }}$ AIAA/CEAS Aeroacoustics Conference and Exhibit, Toulouse, France, May 1998.

[18] Strawn R. C., Oliker L., Biswas R., New Computational Methods for the Prediction and Analysis of Helicopter Noise, Journal of Aircraft 1997;34(5):665-672.

[19] Farassat F., Theory of Noise Generation from Moving Bodies with an Application to Helicopter Rotors, NASA TR R4511, 1975.

[20] Brentner K. S., Numerical Algorithms for Aeroacoustic Integrals with Examples for Rotor Noise Prediction, AIAA Journal 1997;35(4):625-630.

[21] Ianniello S., Algorithm to Integrate the Ffowcs Williams-Hawkings Equation on Supersonic Rotating Domain, AIAA Journal 1999;37(9):1040-1047.

[22] Ianniello S., New perspectives in the use of the Ffowcs Williams-Hawkings equation for aeroacoustic analysis of rotating blades, Journal of Fluid Mechanics 2007;570:79-127.

[23] Colin Y., Caruelle B., Parry A.B., Computational strategy for predicting CROR noise at low-speed. Part III: investigation of the noise radiation with the Ffowcs Williams-Hawkings analogy, AIAA Paper 2012-2223, 18th AIAA/CEAS Aeroacoustics Conference, Colorado Springs, CO, June 2012.

[24] Colin Y., Wlassow F., Caruelle B., Node-Langlois T., Omais M., Spiegel P., Parry A. B., Installation Effects on Contra-Rotating Open Rotor Noise at High-Speed, AIAA Paper 2014-2971, 20th AIAA/CEAS Aeroacoustics Conference, Atlanta, GA, June 2014.

[25] Delattre G., Falissard F., Influence of Torque Ratio on Counter Rotating Open Rotor Interaction Noise, AIAA Paper 2014-2969, 20th AIAA/CEAS Aeroacoustics Conference, Atlanta, GA, June 2014.

[26] Delrieux Y., Overview of rotorcraft acoustic research at Onera, AerospaceLab Journal, Issue 7, June 2014.

[27] Giauque A., Ortun B., Rodriguez B., Caruelle B., Numerical error analysis with application to transonic propeller aeroacoustics, Computer \& Fluids 2012;69:20-34.

[28] Cambier L., Heib S., Plot S., The Onera elsA CFD software: input from research and feedback from industry, Mechanics \& Industry 2013;14(3):159-174.

[29] Kok J. C., Resolving the dependence on freestream values for the k-omega turbulence model, AIAA journal 2000;38(7):1292-1295.

[30] Menter F. R., Two-equation eddy-viscosity turbulence models for engineering applications, AIAA Journal 1994:32(8):1598-1605. 
[31] Delattre G., Falissard F., Aerodynamic and acoustic impacts of a single protuberance placed on the leading edge of the front blades of an open rotor, 48th international Symposium of Applied Aerodynamics of small Bodies and Details, St Louis, France, Mars 2013.

[32] Delattre G., Falissard F., Vion L., Jacquin L., Open rotor interaction noise reduction through front rotor wake modification, International Journal of Aeroacoustics, 2016, 15(1-2):207-227. 